\title{
Transcriptome assembly in Suaeda aralocaspica to reveal the distinct temporal gene/miRNA alterations between the dimorphic seeds during germination
}

Lei Wang ${ }^{1}$, Hong-Ling Wang ${ }^{1}$, Lan Yin ${ }^{2^{*}}$ and Chang-Yan Tian ${ }^{1^{*}}$

\begin{abstract}
Background: Dimorphic seeds from Suaeda aralocaspica exhibit different germination behaviors that are thought to be a bet-hedging strategy advantageous in harsh and unpredictable environments. To understand the molecular mechanisms of Suaeda aralocaspica dimorphic seed germination, we applied RNA sequencing and small RNA sequencing for samples collected at three germination stages.

Results: A total of 79,414 transcripts were assembled using Trinity, of which $57.67 \%$ were functionally annotated. KEGG enrichment unveiled that photosynthesis and flavonol biosynthesis pathways were activated earlier in brown seed compared with black seed. Gene expression analysis revealed that nine candidate unigenes in gibberellic acid and abscisic acid signal transduction and 23 unigenes in circadian rhythm-plant pathway showed distinct expression profiles to promote dimorphic seed germination. 194 conserved miRNAs comprising 40 families and 21 novel miRNAs belonging to 20 families in Suaeda aralocaspica were identified using miRDeep-P and Mfold. The expression of miRNAs in black seed was suppressed at imbibition stage. Among the identified miRNAs, 59 conserved and 13 novel miRNAs differentially expressed during seed germination. Of which, 43 conserved and nine novel miRNAs showed distinct expression patterns between black and brown seed. Using TAPIR, 208 unigenes were predicted as putative targets of 35 conserved miRNA families and 17 novel miRNA families. Among functionally annotated targets, genes participated in transcription regulation constituted the dominant category, followed by genes involved in signaling and stress response. Seven of the predicted targets were validated using $5^{\prime}$ rapid amplification of cDNA ends or real-time quantitative reverse transcription-PCR.
\end{abstract}

Conclusions: Our results indicate that specific genes and miRNAs are regulated differently between black and brown seed during germination, which may contribute to the different germination behaviors of Suaeda aralocaspica dimorphic seeds in unpredictable variable environments. Our results lay a solid foundation for further studying the roles of candidate genes and miRNAs in Suaeda aralocaspica dimorphic seed germination.

Keywords: Suaeda aralocaspica, Euhalophyte, De novo assembly, Transcriptome, Dimorphic seed, Non-dormant, Non-deep dormant, Germination

\footnotetext{
* Correspondence: yinjch@gmail.com; halophyte@gmail.com

${ }^{2}$ ABLife, Inc., Optics Valley International Biomedical Park, Building 18, East

Lake High-Tech Development Zone, 858 Gaoxin Boulevard, Wuhan 430075,

China

${ }^{1}$ State Key Laboratory of Desert and Oasis Ecology, Xinjiang Institute of

Ecology and Geography, Chinese Academy of Sciences, Urumqi 830011

China
} 


\section{Background}

Suaeda aralocaspica is a monoecious annual central Asian halophyte, which is commonly found in salinealkaline sandy soils of Gobi desert. In China, S. aralocaspica is restricted to the inland cold desert of the Junggar Basin, Xinjiang. Desert annuals are known to have welldeveloped seed dispersal and germination mechanisms to survive the harsh environment $[1,2]$. S. aralocaspica produces two distinct types of seeds that differ in morphology, dormancy and germination characteristics [3]. Oblate brown seeds covered with a soft seed coat are highly permeable to water and exhibit non-dormant behavior. Brown seeds can germinate rapidly to high percentages over a wide range of temperature regimes in both white light and darkness. In contrast, elliptical black seeds are covered with a rigid seed coat. Although this type of seeds also take up water, they germinate slowly to low percentages in various viability testing conditions [3]. An imbibed viable seed being not able to germinate under favorable conditions is dormancy [4-7]. We, thereafter, call the black seeds the non-deep dormant seeds [4]. S. aralocaspica developing a unique combination of dispersal and germination strategies via producing dimorphic seeds is thought to be a bethedging strategy advantageous in harsh and unpredictable environments [3, 8-10].

Germination is a critical phase in the plant life cycle. Generally this process starts with the uptake of water by the dry mature seed. Upon imbibition of water, the dry mature seed swell and enzymes and food supplies become hydrated. Hydration re-initiates the metabolic activities in seed to produce energy for growth process. Genome-wide expression studies in Arabidopsis thaliana have been previously applied to gain insight into temporal and spatial changes during Arabidopsis germination and provide important new information about mechanisms controlling germination [11-15]. Nevertheless, a detailed knowledge of the temporal alterations in gene/miRNA in halophyte seed is far missing. In order to understand the control of the timing of germination as well as the underlying molecular processes contributed by the bet-hedging germination, we analyzed $S$. aralocaspica transcriptome by sampling three points along the germination time course. Our high throughput data set will shed light on the temporal alterations occur during dimorphic seed germination of S. aralocaspica and provide a comprehensive list of candidate genes and miRNAs showing potential regulatory mechanisms during this process.

\section{Results}

\section{Morphology of S. aralocaspica seed germination}

In $S$. aralocaspica, seed germination is a process of spiral embryos uncoiling. Black (Bl) dry seeds (DS) had thinly leathery testae, brown $(\mathrm{Br})$ DSs were covered with membranous seed coat (Additional file 1: Figure S1A). At imbibed seed (IS) stage, brown seed absorbed water initially via seed coat and black seed via testae. Along with the imbibition of water, DSs swelled, the seed coat of BrDS stretched while the testa of BIDS cracked (Additional file 1: Figure S1B). At seedling (S) stage, both embryos were uncoiled, the one of brown seed untwisted faster than that of black seed (Additional file 1: Figure S1C).

\section{RNA sequence (RNA-seq) and filter}

A total of 225,861,504 raw reads of 100 bp were generated from BlDS, BlIS, BlS, BrDS, BrIS, and BrS cDNA libraries. After removal of low-quality reads, a total of 201,609,259 high-quality reads were identified, which contained $17,011,844,081$ nucleotides. The average length of the reads is 84 base pairs and the percentage of Q20 bases (base quality more than 20 and an error rate of less than 0.01 ) is $97.43 \%$ (Additional file 2: Table S1 and S2).

\section{De novo transcriptome assembly}

Previous studies have documented that Trinity was a special short-read assembly method for the efficient de novo reconstruction of the transcriptome, and 25-mer was the optimal parameter [16-18]. In this study, the clean reads were assembled de novo using Trinity with a 25-mer parameter and generated 106,171 transcripts. Then, Bowtie 2 [19] was introduced to remove the false positive transcripts, the total transcripts were reduced by 9831. Mapping coverage for this assembly was $83.15 \%$. To reduce the assembly redundancy, we ran the clustering methods using CD-HIT [20] on the assembly. At last, we obtained 79,414 non-redundant transcripts with a total of 51,415,356 nucleotides. The average length and N50 length of these transcripts were $647 \mathrm{bp}$ and $963 \mathrm{bp}$, respectively (Additional file 2: Table S2). The sequencing coverage depth range from 0.9907- to 392,592.2545-fold and the median fold is 16.9680 . Of 79,414 high-quality transcripts, 32,381 (40.77\%) are longer than 500 bp, 15,192 (19.13\%) are longer than $1000 \mathrm{bp}$, and 3151 (3.97\%) are longer than $2000 \mathrm{bp}$.

\section{Functional annotation and coding region sequences (CDS) prediction}

The sequences of $S$. aralocaspica transcripts were searched against the non-redundant $(\mathrm{Nr})$, clusters of orthologous groups (COG), Swiss-Prot and Kyoto Encyclopedia of Genes and Genome (KEGG) protein databases, a total of 44,327 transcripts (55.82\%) were annotated in these four databases (Additional file 2: Table S3). B. vulgaris is the only species that has been sequenced in the family of Chenopodiaceae. Populus euphratica is a halophyte poplar species growing in saline semi-arid areas. There were 44,041 transcripts of $S$. aralocaspica realigned to the B. vulgaris 
genome; and 36,050 transcripts realigned to $P$. euphratica genome (Additional file 2: Table S3). In total, 45,796 transcripts $(57.67 \%)$ were aligned to homologous sequences in public databases, B. vulgaris genome, $P$. euphratica genome. A large proportion had no significant sequence alignment or hits in any of the databases, which suggested that they might contain novel sequences or a high number of special genes in to S. aralocaspica.

Using BLAST2GO [21] program, we obtained gene ontology (GO) functional annotations of the S. aralocaspica transcripts with $\mathrm{Nr}$ annotations. A total of 13,563 transcripts were identified with significant enrichment ( $p$ value $<0.05$ ) (Additional file 3: Figure S2). In the biological process category, "metabolic process", "oxidation reduction", "regulation of transcription, DNA-dependent" were the three dominant subcategories. In the other two main categories, the most prominent subcategories were "ATP binding" and "integral to membrane", respectively.

Based on the four public protein databases, we obtained a total of 36,531 CDSs $(26,017$ CDSs predicted by the BLAST search and 10,514 by ESTScan). As shown in Additional file 4: Figure S3, 39,496 transcripts less than $400 \mathrm{bp}$ were not well annotated. The transcripts not predicted with a CDS were likely either too short to meet the criterion of CDS prediction or were non-coding RNAs.

\section{Identification of differentially expressed genes (DEGs) during seed germination}

To acquire counts data for differential expression analysis, clean reads generated from different stages (DS, IS, S) were mapped to the newly generated reference transcriptome using Bowtie 2 [19]. The reads per kilobase of transcript per million mapped reads (RPKM) value and number of transcripts for each gene were summarized in Additional file 5: Table S4.

Calculated read number was directly used for comparing the differences in gene counts between any two germination stages using EdgeR [22]. As shown in Fig. 1A, more DEGs were identified in black seed (21,327 DEGs) than brown seed (15,827 DEGs) during S. aralocaspica germination (Additional file 6: Table S5). Among them, 8100 DEGs were developmentally regulated in both seed, these DEGs were possibly involved in the common biological processes for both seed during S. aralocaspica germination. The number of up-regulated and downregulated genes in IS vs. DS, S vs. IS, and S vs. DS was displayed in Fig. 1B. Notably, in the comparison between DS and IS stages, the number of DEGs in brown seed was smaller than that of black seed, however, in the comparison between IS and $S$ stages, the number of DEGs in brown seed was dramatically increased and similar to the number of DEGs in black seed. The heatmap of DEGs illustrated that the expression profiles of $\mathrm{BrDS}$ and BrIS were more similar when comparing to the expression profiles of BIDS and BlIS, whereas BIS and $\mathrm{BrS}$ formed a tight cluster with a distinct pattern of gene expression (Fig. 1C). These findings indicated that a larger number of DEGs participated in black seed germination comparing to brown seed, and the difference in the number of DEGs between black and brown seed was most likely attributed to the dormancy breaking required for black seed germination.

KEGG enrichment was performed to identify pathways that were related to $S$. aralocaspica seed germination. Enrichment analysis identified 29 pathways in black seed and 32 pathways in brown seed that were significantly overrepresented ( $p$-value $<0.05$, Fig. $2 \mathrm{~A}$, Additional file 7 : Table S6). There were 18 enriched pathways were overrepresented in both black and brown seed, indicating these 18 KEGG pathways were possibly common biological processes for S. aralocaspica seed germination.

Identification of DEGs between black and brown dry seed To gain an insight into the different biological processes occurred inside the black and brown dry seed, the gene expression levels between BIDS and BrDS were analyzed (Additional file 8: Table S7). In comparison with BrDS, there were 2420 genes up-regulated and 1846 genes down-regulated in BIDS ( $p$-value $<0.01, \mid \log _{2}$ fold change $(F C) \mid \geq 1)$. KEGG enrichment analysis identified 16 significantly overrepresented pathways for up-regulated genes and 17 pathways for down-regulated genes ( $p$-value $<0.05$, Fig. 2B, Additional file 9: Table S8).

\section{The genes involved in gibberellic acid (GA) and abscisic acid (ABA) signal transduction}

The balance of GA: ABA levels and sensitivity have shown to be important factors in the regulation of seed germination [23-25]. In this study, 41 GA or ABA signal genes (57 transcripts) were identified. Among them, 26 unigenes showed differential expression patterns at least in one type of seed germination (Additional file 10: Table S9). Most GA or ABA signal DEGs exhibited similar temporal changes in the expression levels in both seed, indicating these signal DEGs may exert the same functions in black and brown seed germination. However, transcript levels of nine GA or ABA signal genes showed distinct expression patterns between black and brown seed during germination (Fig. 3). The transcript level of PYL2 (Unigene40508) was drastically increased at $\mathrm{S}$ stage in black seed, whereas slightly enhanced in brown seed. The level of PYL12 (Unigene2681) was raised rapidly then reduced markedly in black seed, while continuously dropped to a low level in brown seed. The expressions of PP2CA (Unigene3190) and SLY1 (Unigene49059) in black and brown seed were changed in the opposite directions. The transcript level of OST1 (Unigene28427) was continuously enhanced in black 
a
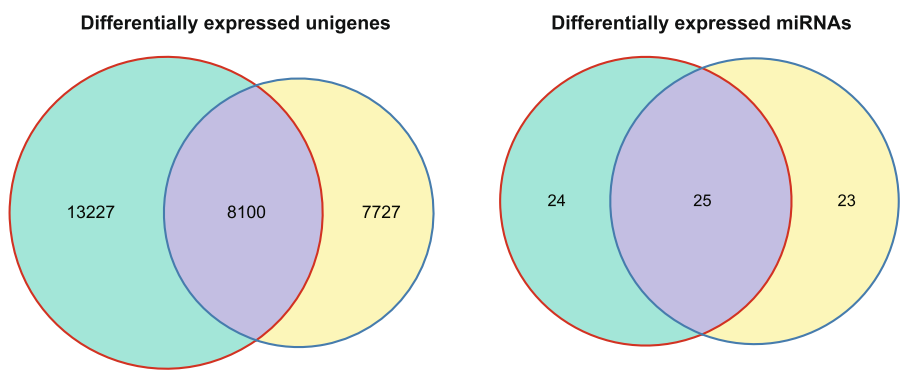

b
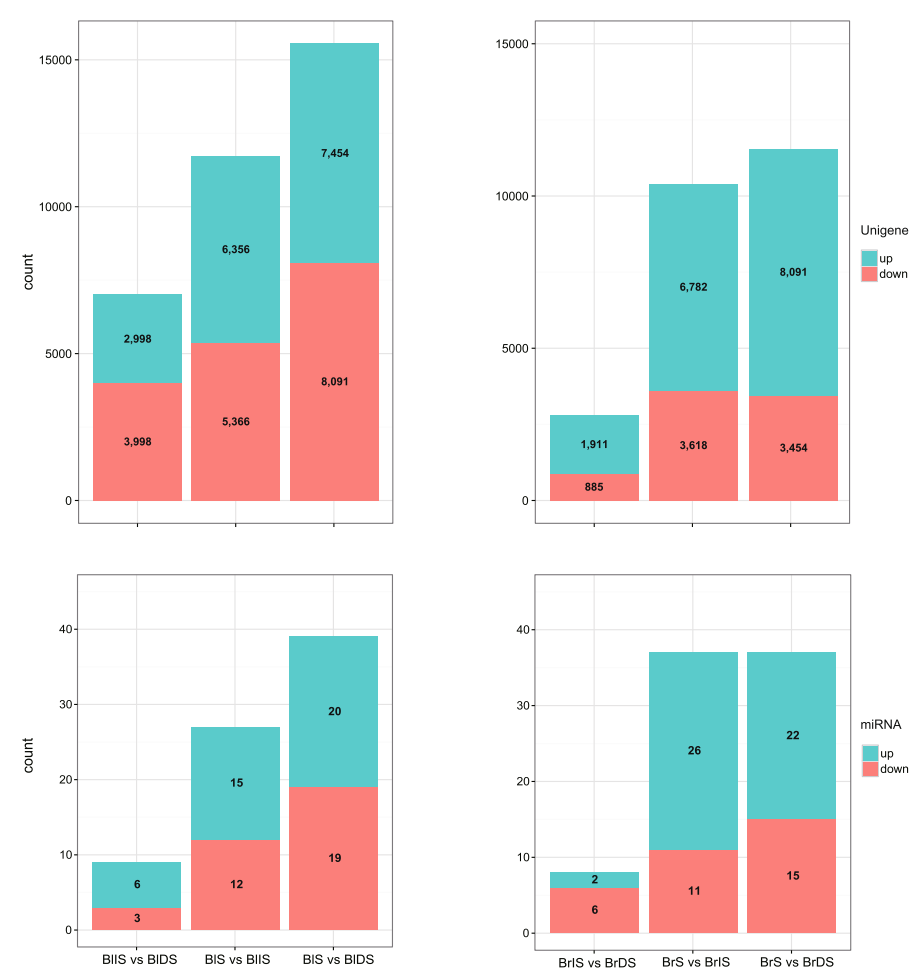

C
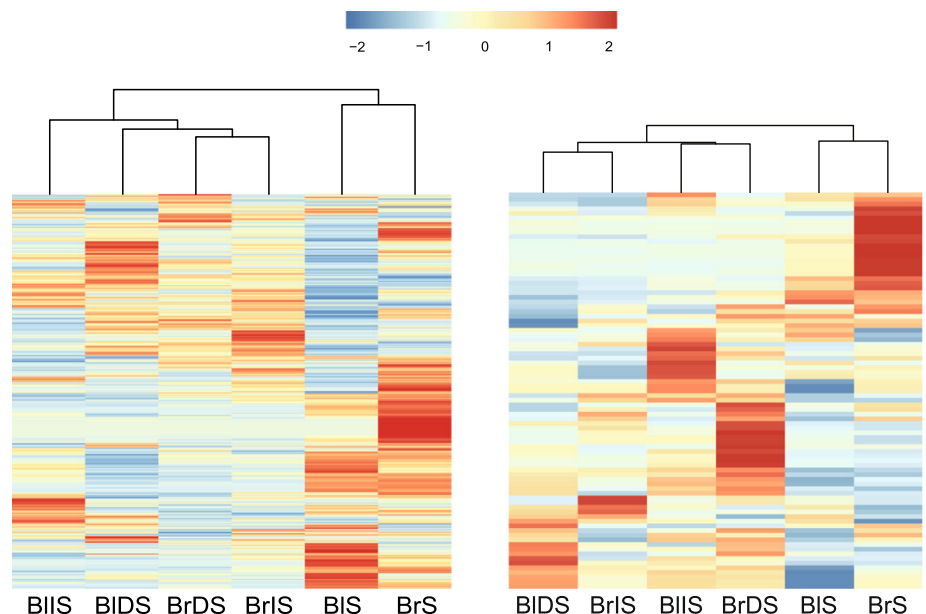

Fig. 1 (See legend on next page.) 
(See figure on previous page.)

Fig. 1 Comparative mRNA and miRNA transcriptome of Suaeda aralocaspica between black and brown seed during germination. a Venn diagram of the number of differentially expressed unigenes (left) and miRNAs (right) in black (green) and brown (yellow) seed germination. $\mathbf{b}$ The number of differentially expressed unigenes (upper) and miRNAs (lower) in black (left) and brown (right) seeds. Aquamarine color bars refer to up-regulated unigenes/miRNAs, salmon color bars refer to down-regulated unigenes/miRNAs. c Expression of the differentially expressed unigenes (left) and miRNAs (right) identified in Suaeda aralocaspica seed germination. BIDS represents black dry seed, BIIS represents black imbibed seed, BIS represents seedlings germinated from black seed, BrDS represents brown dry seed, BrlS represents brown imbibed seed, BrS represents seedlings germinated from brown seed

a

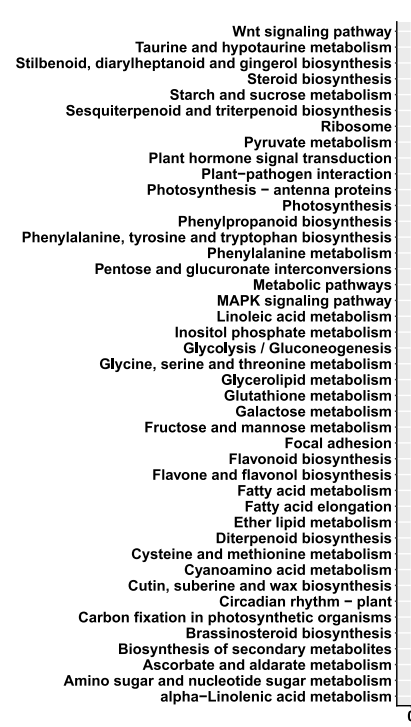

b

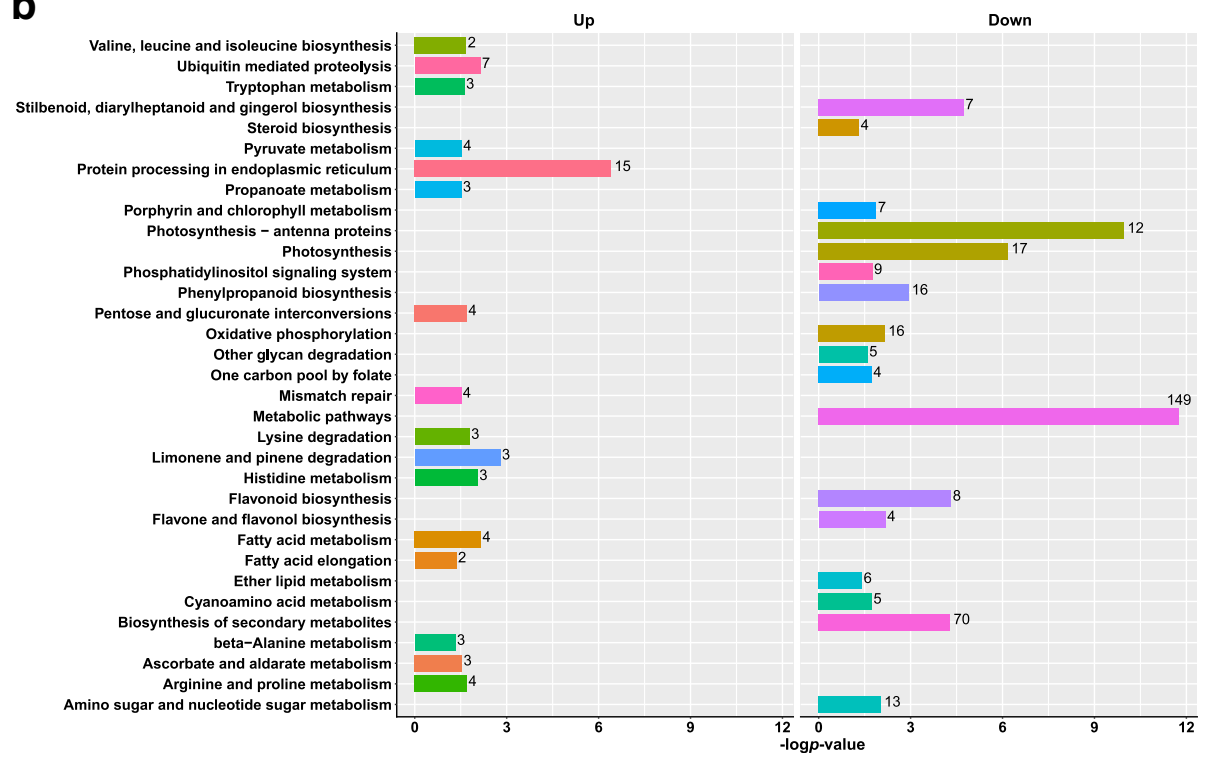

Fig. 2 The significantly overrepresented KEGG pathways identified by enrichment analysis for differentially expressed unigenes. a Differentially expressed unigenes during Suaeda aralocaspica dimorphic seed germination. b Differentially expressed unigenes in the comparison between black and brown dry seed. X-axis represents the base 10 logarithm of the enrichment $p$-value, $y$-axis represents the term of enriched KEGG pathways. The number of differentially expressed unigenes in each pathway is indicated at the end of each bar. Up means up-regulated unigenes in black dry seed, Down means down-regulated unigenes in black dry seed 

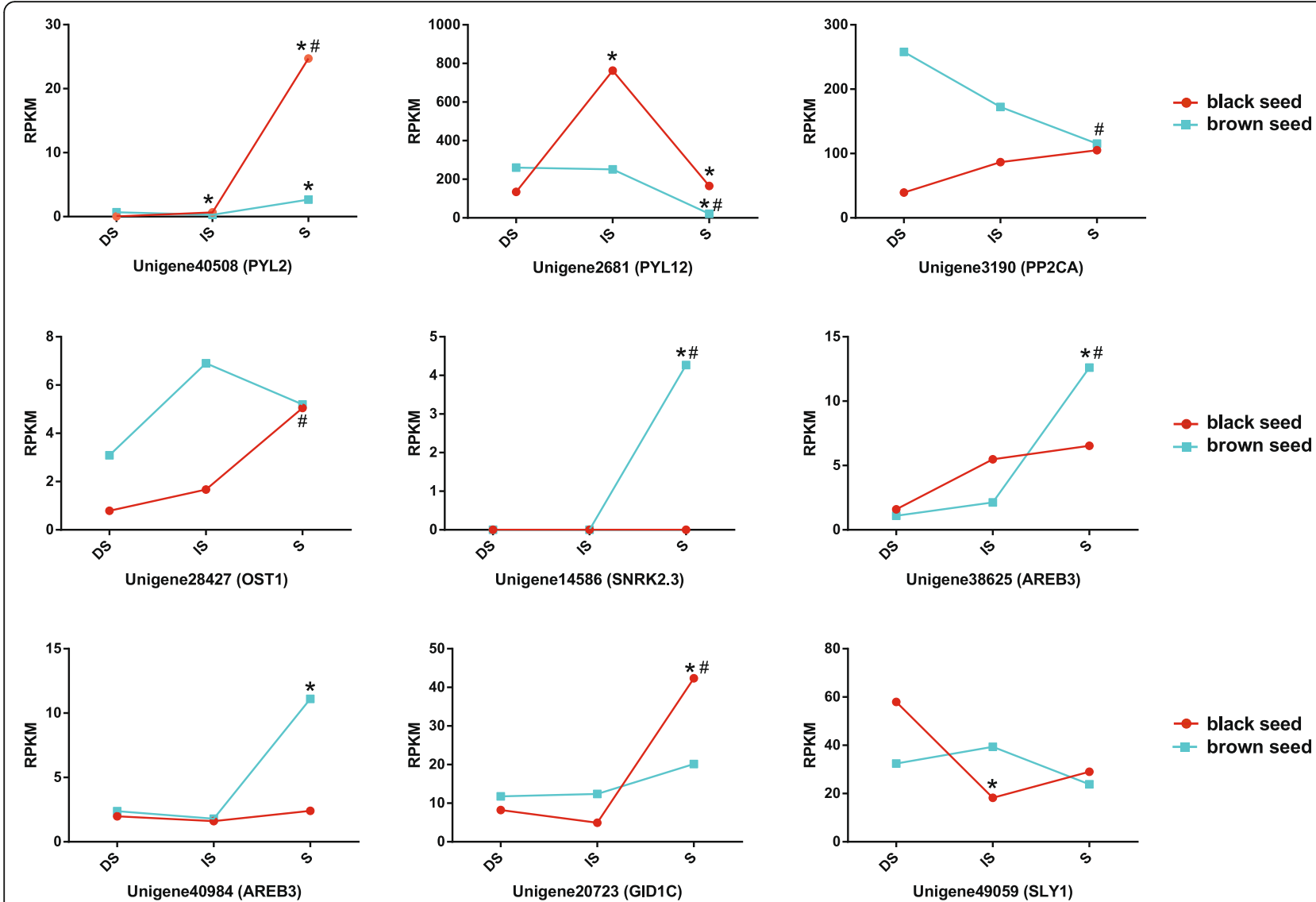

Fig. 3 Distinct expression patterns of abscisic acid and gibberellic acid-related genes between black and brown seed. Red lines represent the transcript levels in black seed, green lines represent the transcript levels in brown seed. The transcript levels were determined by reads per kilobase of transcript per million mapped reads (RPKM). DS represents dry seed, IS represents imbibed seed, $\mathrm{S}$ represents seedlings of Suaeda aralocaspica. * means significant differences $(p<0.05)$ in IS vs. DS or S vs. IS, and \# means significant differences $(p<0.05)$ in $\mathrm{S}$ vs. DS. The abbreviations of abscisic acid/gibberellic acid gene names were listed with parentheses

seed, whereas was increased at first, then decreased in brown seed. The expression of SNRK2.3 (Unigene14586) was only detected at $\mathrm{S}$ stage in brown seed. AREB3 (Unigene38625 and Unigene40984) showed no significant change in expression levels in black seed, but exhibited a dramatic rising at $\mathrm{S}$ stage in brown seed. The level of GID1C (Unigene20723) was slightly decreased then markedly increased in black seed, but gradually enhanced in brown seed.

\section{DEGs involved in plant circadian clock}

Circadian regulation of hormone levels and hormonal signaling modulates many features of plant development, including seed dormancy and germination [26-28]. In this study, KEGG enrichment revealed that 23 DEGs (28 transcripts) were involved in circadian rhythm-plant pathway during black seed germination (Additional file 7: Table S6, Additional file 11: Table S10). As shown in Fig. 4, gene COP1 (Unigene33753, Unigene33751), CHS (Unigene61566, Unigene5107, Unigene48137, Unigene19255),
TOC1 (Unigene45052), and PIF7 (Unigene40139) displayed similar expression patterns in black seed, their expressions were kept at low levels at DS and IS stage, and increased markedly at $\mathrm{S}$ stage. On the contrary, gene SPA2 (Unigene31560), GI (Unigene26154, Unigene26153), SPA1 (Unigene22833, Unigene22832), ZTL (Unigene30382), PHYA (Unigene25752), PRR9 (Unigene25684), and FKF1 (Unigene22119, Unigene22120) were dramatically downregulated at IS stage comparing to DS stage, and their expression were maintained at low levels at IS and $S$ stage. The low expression levels of most of the clock genes at IS stage was possibly related to the dormancy-breaking and germination requirements of black seed. By contrast, in brown seed, only six out of 23 clock genes were developmentally regulated. Gene PIF7 (Unigene40139) and CHS (Unigene5107, Unigene48137) were significantly up-regulated, FKF1 (Unigene22120, Unigene22119) and CHS (Unigene8262) were markedly down-regulated at $\mathrm{S}$ stage when comparing to the other two stages. No 
a

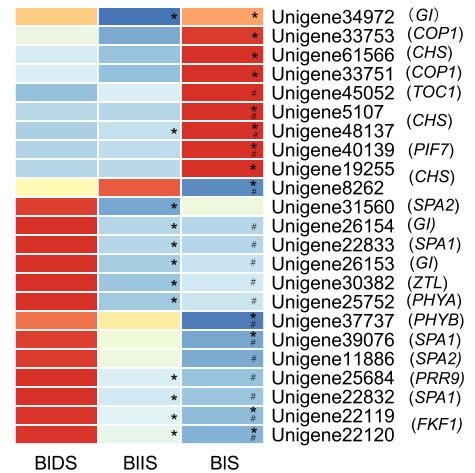

b

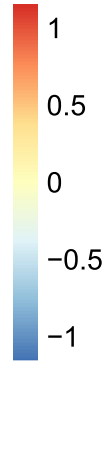

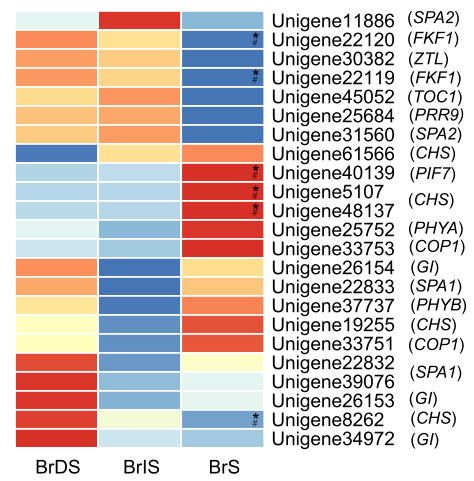

Fig. 4 Heat maps of 23 clock genes in black (a) and brown (b) seed during germination. In the heatmaps, RPKM (reads per kilobase of transcript per million mapped reads) value of each gene was replaced by $\log _{2}$ RPKM. Colors ranged from blue to red, corresponding to low to high expressions. BIDS represents black dry seed, BIIS represents black imbibed seed, BIS represents seedlings germinated from black seed, BrDS represents brown dry seed, BrlS represents brown imbibed seed, BrS represents seedlings germinated from brown seed. ${ }^{*}$ means significant differences $(p<0.05)$ in IS vs. DS or $S$ vs. IS and \# means significant differences $(p<0.05)$ in S vs. DS. The abbreviations of clock gene names were listed with parentheses

statistically significant difference in the expression of clock genes was observed between DS and IS stage in brown seed.

\section{Small RNA sequence (sRNA-seq) analysis}

Although black seed and brown seed share the same set of genes, they are different in many ways, including morphology, dormancy and germination characteristics [3]. These differences may be attributable to the genetic and epigenetic regulations, and in this study we focused on the developmental role of miRNA in S. aralocaspica germination. sRNA-seq generated 5,469,210 to 6,882,624 raw reads, after removing sequences shorter than $18 \mathrm{nt}$ and greater than $30 \mathrm{nt}$ in length, reliable clean reads ranging from 2,483,312 to $3,787,572$ were collected for further analysis (Additional file 2: Table S11). The redundant clean reads were mapped to $S$. aralocaspica mRNA transcriptome database, $43.05 \%$ to $56.32 \%$ redundant sRNAs perfectly matched the S. aralocaspica transcript sequences. The high matching rate may be attributed to insufficient small RNAs. The majority of total sRNA reads ranged from 20 to $24 \mathrm{nt}$ in length (Fig. 5), which are the typical size range of sRNAs generated by Dicer [29]. The major size of sRNA was $24 \mathrm{nt}$ in all six libraries, the second most abundant class was $20 \mathrm{nt}$ in the three libraries of black seed and BrS library, and $23 \mathrm{nt}$ in the libraries of BrDS and BrIS.

\section{Identification of conserved and novel miRNA}

A total of 194 conserved miRNAs were identified comprising 40 miRNA families (Additional file 12: Table

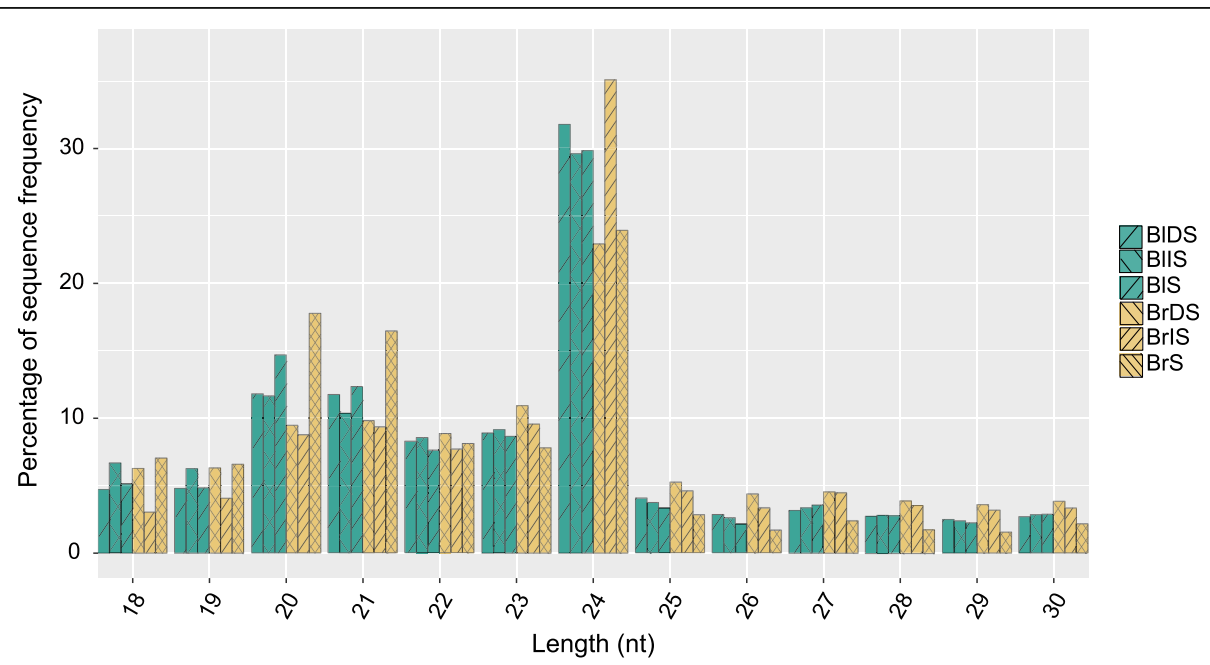

Fig. 5 Length distribution of small RNA sequences in dry seed, imbibed seed, and seedling three libraries. BIDS represents black dry seed, BIIS represents black imbibed seed, BIS represents seedlings germinated from black seed, BrDS represents brown dry seed, BrIS represents brown imbibed seed, BrS represents seedlings germinated from brown seed. nt means nucleotides 
S12), $21 \mathrm{nt}$ and $20 \mathrm{nt}$ were the two major size classes of conserved miRNAs. Among the identified conserved miRNA families, sar-miR156 and sar-miR159 were the two largest families that contained 26 and 28 members, respectively. Whereas, there were 19 miRNA families possessed only one member. The number of members for each family was summarized in Fig. 6. For precursor prediction, the transcript sequences of $S$. aralocaspica were used to determine hairpin structures. Four conserved miRNA precursors with lengths ranging from $87 \mathrm{nt}$ to $171 \mathrm{nt}$ were identified. Their minimal folding free energy indices (MFEIs) varied from 0.88 to 1.28 with an average of 1.09 , which was consistent with that revealed in other plant miRNAs [30]. The total counts of conserved miRNAs were lowest in BlIS library and were rapidly increased in BIS library. In contrast, the total counts of conserved miRNAs were slightly increased in BrIS library compared to BrDS and reached the highest in BrS library (Additional file 12: Table S12). This finding indicated that the expression of conserved miRNAs in black seed was suppressed at IS stage.

We identified 22 putative novel miRNAs belonging to 20 families in S. aralocaspica and named them as sarmiR1 to sar-miR20 (Additional file 13: Table S13). Among the identified novel miRNAs, sar-miR13 was found to be homologous to sar-miR159 family members with one mismatch, therefore sar-miR13 was classified as a member of miR159 family. Sar-miR1a and sarmiR1b shared similar mature sequence, and sar-miR6a and sar-miR6b were homologous with each other, thereafter sar-miR1a and sar-miR1b were classified into sar-
miR1 family, and sar-miR6a and sar-miR6b were classified into sar-miR6 family. The length of mature sequences of the novel miRNAs varied from $18 \mathrm{nt}$ to $24 \mathrm{nt}$, and $21 \mathrm{nt}$ was the major size class. By counting reads mapped to the different novel miRNAs in each sample, we found most novel miRNAs had relatively low expression levels, which was consistent with the feature of species-specific miRNAs. The length of novel miRNA precursors ranged from $61 \mathrm{nt}$ to $170 \mathrm{nt}$, and MFEIs varied from 0.52 to 1.77 with an average value of 1.03 . The total counts of novel miRNAs displayed a change trend similar to that of conserved miRNAs (Additional file 13: Table S13), suggesting that the expression of novel miRNAs in black seed was also suppressed at IS stage.

The precursor sequences and secondary hairpin structures of S. aralocaspica conserved and novel miRNAs predicted by Mfold [31] were represented in Additional file 14: Table S14 and Additional file 15: Figure S4.

\section{miRNA expression profiles}

There were 49 miRNAs in black seed and 48 miRNAs in brown seed differentially expressed (DE) during dimorphic seed germination ( $p$-value $<0.01$ and $\left|\log _{2} \mathrm{FC}\right|$ 21) (Fig. 1A, Additional file 16: Table S15). In the comparison of miRNA expression profiles between the three consecutive stages, we found that the numbers of $\mathrm{DE}$ miRNAs were dramatically increased in both seed at the phase transition from IS to $S$ comparing to those at the phase transition from DS to IS, and the number of upregulated miRNAs at the phase transition from IS to $S$ in brown seed was much larger than that in black seed

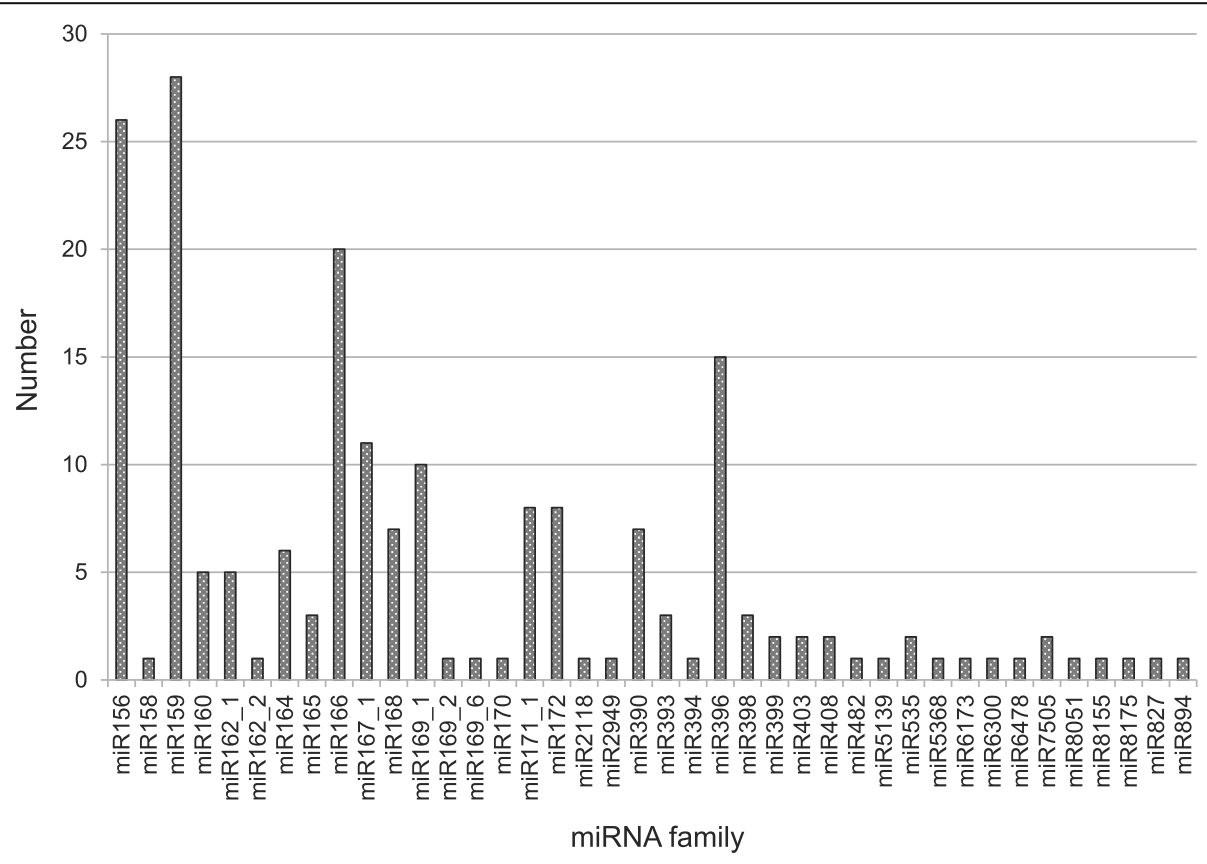

Fig. 6 The number of members in each conserved miRNA family in Suaeda aralocaspica 
(Fig. 1B). Hierarchical cluster analysis illustrated that the expression profiles of DE miRNAs at DS and IS stages were similar for both seeds, DE miRNAs at S stage displayed a distinct expression pattern (Fig. 1C). These findings suggested that DE miRNAs majorly functioned in the phase transition from IS to $S$, and a larger number of miRNAs in brown seed took part in this transition process than black seed.

Based on their expression patterns, DE miRNAs were divided into four categories (Table 1). The first category contained miRNAs that were up-regulated during seed germination. The second category comprised miRNAs that were down-regulated during seed germination. The third category was composed of miRNAs that had relative low levels in dry seed, were up-regulated in imbibed seed then down-regulated in seedling. The last category contained miRNAs that were down-regulated in IS vs. DS, then up-regulated in S vs. IS. Among DE miRNAs, 43 conserved and nine novel miRNAs showed distinct expression patterns between black and brown seed during germination.

\section{Target prediction of conserved and novel miRNAs}

The 79,414 assembled transcripts from S. aralocaspica mRNA transcriptome database were used as a custom target database, 194 conserved and 22 novel mature miRNAs were used as a custom miRNA database. Using TAPIR [32], a total of 170 unigenes were predicted as potential targets of 35 conserved miRNA families (Additional file 17: Table S16). Thirty-three unigenes (19.41\%) were homologous to the previously confirmed or predicted targets of the same miRNA families in Arabidopsis thaliana, Oryza sativa or Solanum lycopersicum (Table 2). Most of these conserved targets (23 out of 33) encoded essential transcription factors, and the rest of targets were homologs of Dicer protein, F-box protein, copper/zinc superoxide dismutase, inorganic phosphate transporter, plantacyanin, laccase, and CC-NBS-LRR protein. There were 137 putative targets of conserved miRNAs were not conserved in other plant species. Of these target genes, 76 (44.7\%) targets presented no function annotation. The annotated 94 unigenes were classified into 16 categorizes according to their molecular and biological functions (Fig. 7A). Genes involved in transcription regulation $(31,33 \%$; including 26 transcription factors) comprised the most dominant category, followed by unigenes involved in the other two main categories, signaling $(15,16 \%)$ and stress response $(12,13 \%)$. In the same way, 42 unigenes were identified as putative targets of 17 novel miRNA families and one miR159 family member (Additional file 18: Table S17). Most of these target genes $(28,66.67 \%)$ were not functionally annotated. The rest annotated targets were involved in eight categories (Fig. 7B). Unigenes involved in stress response (3, 22\%), signaling $(2,14 \%)$, transcription regulation (2, $14 \%)$, energy (2, 14\%), and RNA processing (2, 14\%) accounted for the major proportions.

Due to insufficient $S$. aralocaspica mRNA sequences, we were not able to predict the targets for five conserved miRNA families and two novel miRNA families.

\section{Validation of miRNA targets by 5 ' rapid amplification of CDNA ends (RACE)}

To validate the results of in silicon analysis, we amplified the predicted target genes through $5^{\prime}$ RACE-PCR. The cleavage sites in four predicted target genes of $S$. aralocaspica were successfully detected (Fig. 8). Unigene24713, Unigene44608, Unigene47858, and Unigene19904 were

Table 1 Differentially expressed Suaeda aralocaspica miRNAs during seed germination

\begin{tabular}{l} 
Black seed \\
\hline Up-regulated \\
sar-miR156t-5p, sar-miR159k, sar-miR160a, sar-miR164b, sar-miR166g, \\
sar-miR168d, sar-miR169a, sar-miR169i, sar-miR171c, sar-miR172a, \\
sar-miR172h, sar-miR393b, sar-miR396a, sar-miR396d, sar-miR396e, \\
sar-miR396j, sar-miR396k, sar-miR396o, sar-miR6300a, sar-miR6b, \\
sar-miR10 \\
Down-regulated \\
sar-miR157a, sar-miR319d, sar-miR319h, sar-miR166b-3p, sar-miR166d, \\
sar-miR166l, sar-miR166r, sar-miR167b, sar-miR167c, sar-miR167d, \\
sar-miR167e, sar-miR167f, sar-miR167h, sar-miR167i, sar-miR167j, \\
sar-miR171e-3p, sar-miR172b, sar-miR172c, sar-miR172e, sar-miR398c, \\
sar-miR894a, sar-miR2, sar-miR7, sar-miR11, sar-miR12, sar-miR14, \\
sar-miR16 \\
Up-regulated then down-regulated \\
sar-miR5 \\
Down-regulated then up-regulated
\end{tabular}
Brown seed

sar-miR319d, sar-miR160a, sar-miR164a, sar-miR164b, sar-miR166g, sar-miR167e, sar-miR169a, sar-miR171a, sar-miR171c, sar-miR172a, sar-miR172h, sar-miR390b, sar-miR396a, sar-miR396d, sar-miR396e, sar-miR396f, sar-miR396h, sar-miR396i, sar-miR396j, sar-miR396k, sar-miR396m, sar-miR396o, sar-miR398c, sar-miR6a, sar-miR10

sar-miR156n, sar-miR162b, sar-miR169d, sar-miR171h, sar-miR172b, sar-miR172c, sar-miR5139a, sar-miR5368a, sar-miR6173a, sar-miR6478a, sar-miR8155a, sar-miR8175a, sar-miR894a, sar-miR7, sar-miR11, sar-miR14, sar-miR17, sar-miR18, sar-miR19

sar-miR159a, sar-miR159j 
Table 2 Conserved $^{a}$ miRNA targets identified in Suaeda aralocaspica

\begin{tabular}{|c|c|c|}
\hline miRNA family & Target & Annotation \\
\hline \multirow[t]{2}{*}{ miR156/157 } & Unigene25708 & squamosa promoter-binding-like protein 6 isoform $\times 2$ \\
\hline & Unigene30985 & squamosa promoter-binding-like protein 2 \\
\hline miR159 & Unigene5343 & MYB protein, DUO POLLEN 1 \\
\hline \multirow[t]{3}{*}{ miR160 } & Unigene37068 & auxin response factor 10 \\
\hline & Unigene36784 & auxin response factor 16 \\
\hline & Unigene39132 & auxin response factor 17 \\
\hline miR162 & Unigene29941 & SUSPENSOR 1, Dicer-like 1 \\
\hline miR164 & Unigene38871 & NAC domain containing protein 100 \\
\hline \multirow[t]{2}{*}{$\mathrm{miR} 165 / 166$} & Unigene32963 & member of HD-ZIP III family, INCURVATA 4 \\
\hline & Unigene47107 & member of HD-ZIP III family, REVOLUTA \\
\hline \multirow[t]{3}{*}{ miR169 } & Unigene44960 & CCAAT binding factor-HAP2-like protein, nuclear factor Y subunit A1 \\
\hline & Unigene26545 & CCAAT binding factor-HAP2-like protein, nuclear factor Y subunit A9 \\
\hline & Unigene25577 & nuclear factor $Y$ subunit A-3 \\
\hline \multirow[t]{2}{*}{$\operatorname{miR170/171~}$} & Unigene34442 & HAIRY MERISTEM 3, SCL6-IV \\
\hline & Unigene44608 & HAIRY MERISTEM 4, SCL15 \\
\hline \multirow[t]{2}{*}{ miR172 } & Unigene26235 & TARGET OF EARLY ACTIVATION TAGGED 1 \\
\hline & Unigene13804 & AP2-like ethylene-responsive transcription factor TOE3 \\
\hline \multirow[t]{2}{*}{ miR319 } & Unigene42701 & TEOSINTE BRANCHED 1, cycloidea and PCF transcription factor 2 \\
\hline & Unigene46584 & TEOSINTE BRANCHED 1, cycloidea and PCF transcription factor 3 \\
\hline \multirow[t]{3}{*}{ miR393 } & Unigene37071 & F-box protein, TRANSPORT INHIBITOR RESPONSE 1 \\
\hline & Unigene37070 & F-box protein, TRANSPORT INHIBITOR RESPONSE 1 \\
\hline & Unigene47621 & Auxin signaling F-box 3 \\
\hline miR394 & Unigene26101 & a putative F-box protein, LEAF CURLING RESPONSIVENESS \\
\hline \multirow[t]{5}{*}{ miR396 } & Unigene44576 & growth-regulating factor 2 \\
\hline & Unigene20645 & growth-regulating factor 3 \\
\hline & Unigene24713 & growth-regulating factor 4 \\
\hline & Unigene44047 & growth-regulating factor 5 \\
\hline & Unigene20255 & growth-regulating factor 7 \\
\hline miR398 & Unigene2632 & copper/zinc superoxide dismutase 1 \\
\hline miR399 & Unigene40813 & a ubiquitin-conjugating E2 enzyme, UBIQUITIN-CONJUGATING ENZYME 24 \\
\hline \multirow[t]{2}{*}{ miR408 } & Unigene47976 & plantacyanin \\
\hline & Unigene39882 & laccase 3 \\
\hline miR482 & Unigene39969 & disease resistance protein, CC-NBS-LRR class \\
\hline
\end{tabular}

${ }^{a}$ Conserved with Arabidopsis thaliana, Oryza sativa, or Solanum lycopersicum

confirmed to be targets of sar-miR396d, sar-miR170/171, sar-miR169i, and sar-miR5, respectively. Sequencing of the sar-miR169i-cleaved 5' RACE product of Unigene 47858 identified a precise slice between the nucleotides 10 and 11 in the complementary region of the miRNA: mRNA pair (Fig. 8). Unigene47858 encodes a protein homologous to the Arabidopsis late embryogenesis abundant (LEA) protein family protein. Unigene44608 and Unigene19904 were validated to be targets of sar-miR170/171 and sar-miR5, respectively, with multiple cleavage sites. Unigene44608 is homologous to an Arabidopsis protein coded by GRAS family transcription factor, and Unigene19904 is homologous to an Arabidopsis protein coded by protein kinase superfamily protein. Unigene24713, the putative target of sar-miR396d, was also evaluated for its cleavage site. Unigene24713 encodes a protein homologous to the Arabidopsis transcription factor growth-regulating factor 4. A shorter or longer cleaved sequence was observed for the four putative targets after $5^{\prime}$ RACE analysis, which could be attributed to secondary siRNA in the 21-nucleotide register with the cleavage site for miRNAs as previously documented [33-35]. 


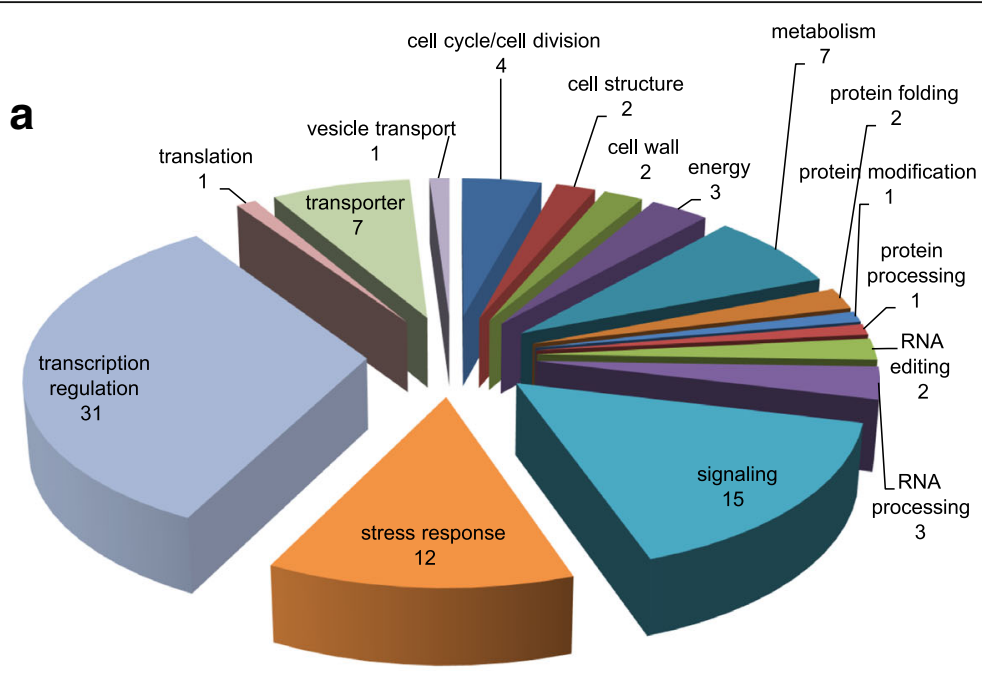

b

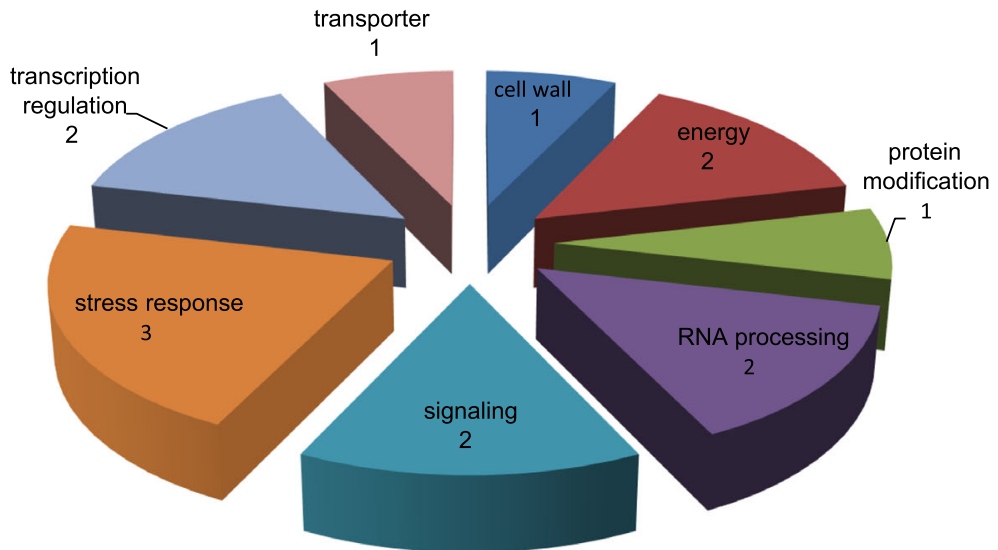

Fig. 7 Functional classifications of predicted targets of conserved (a) and novel (b) miRNAs in Suaeda aralocaspica. Only the functionally annotated target genes are shown. The number of targets in each category is shown under the iterm

\section{Validation of the expression profiles of unigenes and miRNAs by real-time quantitative reverse transcription (qRT)-PCR}

To validate the expression of identified unigenes and $\mathrm{miR}$ NAs from transcriptome analysis, six GA or ABA signal genes (Unigene20723, Unigene49059, Unigene2681, Unigene3190, Unigene28427, and Unigene38625), five conserved miRNAs (sar-miR166l, sar-miR166r, sar-miR169i, sar-miR394a and sar-miR396e) and their potential targets (Unigene32963, Unigene47858, Unigene26101, Unigene44047), and three novel miRNAs (sar-miR5, sar-miR7 and sar-miR17) were selected and subjected to the qRTPCR analysis. For calculating the relative expression of each unigene or miRNA, the $\mathrm{Ct}$ value at DS stage was used as a reference. Analysis of transcript levels by qRTPCR showed that the ten unigenes $\left(R^{2}=0.459, P<0.01\right)$ and eight miRNAs $\left(R^{2}=0.402, P<0.01\right)$ all displayed positive correlation between the RNA/sRNA-seq quantitative measurement and qRT-PCR method (Additional file 19: Figure S5, Additional file 20: Table S18), suggesting that most of the tested unigenes and miRNAs showed similar developmental alterations in the two methods and our high-throughput data were reliable.

Further, we assessed the expression patterns of five miRNAs and their putative targets using real-time qRTPCR. As shown in Additional file 21: Figure S6, the expression of sar-miR166l and sar-miR166r was suppressed while the level of Unigene32963 was increased during black seed germination. The level of sar-miR169i was enhanced while the expression of Unigene47858 was inhibited at BrS stage. The expression of sar-miR394a was decreased, whereas, the level of Unigene26101 was elevated at BIS stage. The level of sar-miR396e was increased while the expression of Unigene44047 was repressed at both BIS and BrS. The putative target genes exhibited opposite expression patterns to their 

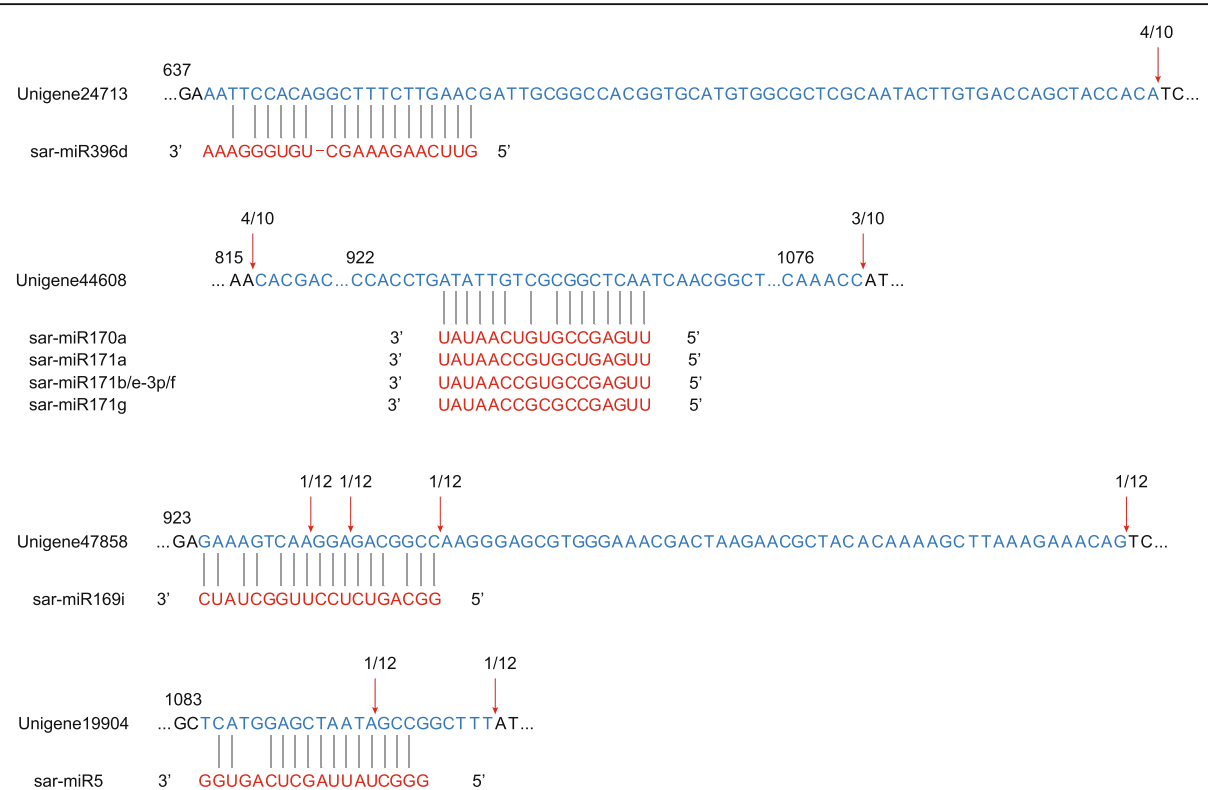

Fig. 8 Validation of miRNA-guided target unigene cleavage. Partial sequences from target genes were aligned with the corresponding miRNAs, Each top strand (blue) represents a miRNA-homologous site in the target gene and each bottom strand (red) represents the aligned sequence of miRNA. Red arrows indicate the observed miRNA cleavage sites following 5' RACE analysis, with the frequency of clones shown

corresponding miRNAs at certain time points of germination, suggesting that these targets may be regulated post-transcriptionally by the action of miRNAs.

\section{Discussion}

This study provided an overview of the genes and miRNAs presented in a non-model euhalophyte species and identified the candidates associated with the germination process of dimorphic seed under the control of a bethedging strategy.

\section{The metabolic processes activated earlier in brown seed than in black seed during S. aralocaspica seed germination}

It is documented that mature seeds possess photosynthetically active chloroplasts, which maintain photosynthetic activity during the period of reserve accumulation, contributing oxygen supply and coupled biosynthetic fluxes [36-38]. Morphologically, the membranous seed coat of $S$. aralocaspica brown seed (Additional file 1: Figure S1A) would allow the embryo gain more amount of light than the black seed embryo, which is regarded as a critical factor for seed photosynthesis. In order to comprehensively understand the initial important metabolic processes activated at the transition from dry seed to germination, we combined the KEGG enrichment results of DEGs in dry seeds and during seed germination. We found that some KEGG pathways were activated in both seed germination, but they were activated earlier in brown seed compared with black seed. For instance, "Photosynthesis-antenna proteins", "Photosynthesis", and "Flavone and flavonol biosynthesis" pathways were activated at DS stage in brown seed (Fig. 2B, Additional file 9: Table S8), but activated at IS and S stages in black seed (Fig. 2A, Additional file 7: Table S6). Among 12 antenna proteins and 17 photosynthesis proteins that were up-regulated at DS stage in brown seed compared with black seed, 12 antenna proteins and 15 photosynthesis proteins were up-regulated at the late stages of germination in black seed (Fig. 2, Additional file 22: Table S19, Additional file 23: Table S20). This finding provided strong evidence that photosynthesis process was activated earlier in brown seed than black seed during $S$. aralocaspica germination. Flavonols have been shown to negatively regulate auxin transport and dependent physiological processes [3943]. Auxin gradient is crucial for de novo induction and maintenance of root meristematic activity [44-46]. Flavonol biosynthesis is induced by auxin [42], and the expression of genes involved in this pathway is light dependent $[47,48]$. In $S$. aralocaspica, the activation of flavonol biosynthesis at DS stage in brown seed demonstrated that seedling establishment could be initiated earlier in brown seed than in black seed. Additionally, miR393 targets F-box genes that encode auxin receptors [49-51], and TRANSPORT INHIBITOR RESPONSE 1 (TIR1) acts as an auxin receptor mediating transcriptional responses to auxin [52]. In this study, Unigene37070 and Unigene37071, encoding proteins homologous to TIR1, were predicted as targets of sar-miR393b (Table 2, Additional file 17: Table S16). sarmiR393b was up-regulated at $S$ stage comparing to the other two stages in black seed (Table 1, Additional file 16: Table S15), while maintained at a high level in brown seed. Unigene37070 and Unigene37071 showed opposite 
expression patterns to sar-miR393b (Additional file 5: Table S4) during germination. These findings suggested that the distinct expression patterns of miR393 and its targets between black and brown seed maybe associated with the early activation of flavonol biosynthesis in brown seed.

\section{Candidate $A B A$ and GA genes that may contribute to the} diversity in seed germination behaviors in S. aralocaspica Protein phosphatase 2CA (PP2CA) is generally upregulated in response to ABA in Thellungiella salsuginea [53] and salt treatment in Suaeda fruticosa [54]. In S. aralocaspica, the expression of PP2CA (Unigene3190) was gradually increased in black seed but continuously decreased in brown seed (Fig. 3, Additional file 10: Table S9), suggesting that PP2CA may play a role in stress response during black seed germination. In Arabidopsis, three subclass III SNF1related kinase $2 \mathrm{~s}$ (SnRK2s), SRK2D/SnRK2.2, SRK2E/ SnRK2.6/OST1 and SRK2I/SnRK2.3 (SRK2D/E/I), are strongly activated by ABA and osmotic stresses $[55,56]$. At present study, one homologous of OST1 (Unigene28427) was up-regulated in black seed but maintained at a relative high level in brown seed (Fig. 3, Additional file 10: Table S9), implying that OST1 may be activated earlier in brown seed than black seed, and it could be involved in ABA and osmotic stress response in both seed during S. aralocaspica germination process. One homologous of SnRK2.3 (Unigene14586) was only detected at $\mathrm{S}$ stage in brown seed (Fig. 3, Additional file 10: Table S9), suggesting this gene may play a role in stress tolerance in brown seed during postgermination growth. GA promotes seed germination by enhancing the proteasomal destruction of ral guanine nucleotide dissociation stimulator-like 2 (RGL2) [57], and F-box protein SLEEPY1 (SLY1) is required for this process [58]. In this study, SLY1 showed different expression trend between black and brown seed (Fig. 3, Additional file 10: Table S9), indicating that GA signaling maybe regulated differently during dormant and non-dormant seed germination. Further, there were several receptors and downstream transcription factors of ABA or GA signaling, including PYL2 (Unigene40508), PYL12 (Unigene2681), AREB3 (Unigene38625 and Unigene40984), and GID1C (Unigene20723), showed distinct expression patterns between black and brown seed during germination (Fig. 3, Additional file 10: Table S9). This difference may contribute to the different germination behaviors of S. aralocaspica dimorphic seeds in order to cope with the harsh and unpredictable environment. Further investigation on the molecular mechanisms underlying the dimorphic seed germination controlled by GA and ABA signaling is needed.

\section{Candidate clock genes that may contribute to dormancy breaking during black seed germination}

Using real-time RT-PCR, Arabidopsis circadian clock has been demonstrated to act as an important signal integrator regulating dormancy release [26]. In current study, the high throughput data allowed us to comprehensively compare the dynamic expression changes occurred in dormant seed and non-dormant seed during germination in the same species. KEGG enrichment identified 23 clock genes participated in the circadian rhythm pathway in S. aralocaspica black seed (Additional file 11: Table S10). These 23 genes exhibited the well-characterized transcriptional circadian rhythms of dormancy breaking. The transcript levels of SPA, GI, ZTL, PHYA, PRR9, and FKF1 were high and the transcript levels of COP1, CHS, TOC1, and PIF7 were relatively low in non-deep dormant dry seed (Fig. 4). At IS stage, seed imbibition and testa rupture had a general suppression effect on the gene expressions of GI, CHS, $S P A, Z T L, P H Y A, P R R 9$, and FKF1, indicating the clock components in imbibed black seeds may be responding to the environmental and endogenous signals for dormancy-breaking requirement. At $\mathrm{S}$ stage, uncoiled embryo rupture promoted the expressions of GI, COP1, CHS, TOC1, and PIF7, which possibly initiated the seedling establishment of circadian oscillations in black seed. In contrast, brown seed exhibited a different transcriptional profile of these 23 clock genes and the circadian rhythms seemed to persist during the whole germination process, which was consist with its genotype that seedling establishment-related biological process initiated early at dry seed stage (Fig. 2B, Additional file 9: Table S8).

\section{The transcriptional suppression of miRNAs at IS stage in black seed}

Similar to the transcriptional suppression of clock genes at BlIS stage, we found the total counts of conserved and novel miRNAs were lowest at IS stage in black seed comparing to the other two stages (Additional file 13: Table S13, Additional file 14: Table S14), implying that the expression levels of miRNAs could be suppressed by dormancy breaking either. We speculated that transcriptional suppression maybe one crucial success factor for non-deep dormant seed to break dormancy. In plants, DICER-LIKE 1 (DCL1) is the main processor in miRNA biogenesis [59] and subject to negative feedback regulation through miR162 [60]. At present study, we predicted that sar-miR162b targeted one DCL1 protein (Table 2, Additional file 17: Table S16). In black seed, the expression of sar-miR162b was kept at the same level at DS and IS, then was decreased at S stage (Table 1, Additional file 16: Table S15). In brown seed, the expression level of sar-miR162b was relatively high at DS, then decreased at IS and further decreased at S. This finding indicated that miR162 could play an important role in transcriptional suppression of miRNAs and dormancy breaking through its target DCL1. 
miRNAs with distinct expression patterns between the dimorphic seeds and their roles in S. aralocaspica seed germination

The germination process is a coordinated action of multiple environmental responsive genes, which also crosstalk with other components of the elaborate hormone signaling networks. miRNAs play critical roles in the regulation of these biological processes [61, 62]. Arabidopsis miR156 is essential for vegetative leaf development from the cotyledon-stage seedlings by downregulating its target SPL $[63,64]$. Maize miR156 is differentially down-regulated at imbibition step during seed germination [65]. In this study, sar-miR156t-5p was upregulated in black seed during germination (Table 1, Additional file 16: Table S15), whereas, no significant sar-miR156t-5p expression difference was identified between the germination stages in brown seed. Two SPL proteins were predicted as the targets of sar-miR156t-5p (Table 2, Additional file 17: Table S16), implying miR156 could play important roles in vegetative leaf development from black seed seedling by regulating SPL, while brown seed may depend less on this mechanism in vegetative leaf development after germination. miR159 is induced by ABA, suppresses MYB33 and MYB101 transcript levels and renders plants hyposensitive to ABA during germination [66]. miR159 was also induced under drought conditions suggesting it maybe a signal factor in sensing the environment surrounding a seed to ensure plant survival after germination $[62,66]$. At present study, sar-miR159k was up-regulated in black seed, while sar-miR159j were up-regulated in IS vs. DS but down-regulated in S vs. IS in brown seed during germination (Table 1, Additional file 16: Table S15). One MYB protein was predicted to be the target of sarmiR159j and sar-miR159k (Table 2, Additional file 17: Table S16), indicating that miR159-mediated regulatory module may be linked with ABA responses and homeostasis during $S$. aralocaspica seed germination, and desensitize hormone signaling to tolerate adverse environments in black seed during post-germination growth. SUPPRESSOR OF MAX2 1 (SMAX1) is an important component of karrikins (KAR) / strigolactone (SL) signaling, regulating germination and hypocotyl elongation [67]. In this study, we predicted that sar-miR167e targeted a homolog of SMAX1 (Additional file 17: Table S16). sar-miR167e was down-regulated in black seed but up-regulated in brown seed during germination (Table 1, Additional file 16: Table S15), suggesting miR167 may regulate germination and hypocotyl elongation in different ways between black and brown seed. miR171targeted scarecrow-like (SCL) 6/22/27 proteins mediate GA-DELLA signaling in the coordinate regulation of chlorophyll biosynthesis under light conditions [68]. SCL15 plays an essential role in repressing embryonic traits in Arabidopsis seedlings [69]. At present study, sar-miR171e-3p was down-regulated in black seed in IS vs. DS, and sar-miR171a was up-regulated and sarmiR171h was down-regulated in brown seed during germination (Table 1, Additional file 16: Table S15). A homolog of SCL6-IV was predicted as the target of sar$\mathrm{miR} 171 \mathrm{a} / \mathrm{e}-3 \mathrm{p} / \mathrm{h}$, and a homolog of protein of SCL15 was predicted to be the target of sar-miR171a/e-3p (Table 2, Additional file 17: Table S16), implying that miR171-SCL module may fine-tune the GA-regulated chlorophyll biosynthesis and participate in the regulation of embryo-to-seedling phase transition during S. aralocaspica seed germination.

miRNAs also play an important role in various stress responses. miR169 is induced by drought [70] and high salinity [71] in rice, but repressed by salt in Thellungiella salsuginea [72]. miR169 targeted NF-YA encodes a subunit of the NF-Y complex transcription factor which is involved in root development, nitrogen-starvation responses, and plant responses to drought and salt stresses $[71,73,74]$. LEA proteins have crucial roles in cellular dehydration tolerance [75]. At present study, sarmiR169i was up-regulated in black seed, while sarmiR169d was down-regulated in brown seed during germination (Table 1, Additional file 16: Table S15). We predicted that sar-miR169d targeted one NF-YA transcription factor and sar-miR169i targeted one LEA protein (Table 2, Additional file 17: Table S16), indicating that miR169 may exert diverse roles in response to drought and salt stresses during $S$. aralocaspica germination. Phosphatase 2C (PP2C) family proteins are key players in ABA signal transduction during seed germination $[24,76]$. Highly ABA-induced PP2C gene 2 (HAI2) is a member of the $\mathrm{PP} 2 \mathrm{C}$ family and recently found to be also involved in ABA-independent drought-associated signaling [77]. One FK506-Binding Protein (FKBP) family protein, ROF1, is reported to play an important role in salt stress responses during Arabidopsis seed germination [78]. In this study, sar-miR172e was downregulated in black seed during germination (Table 1, Additional file 16: Table S15), whereas, no significant difference in sar-miR172e expression was identified between the germination stages in brown seed. A homolog of HAI2 and one FKBP protein were predicted to be the targets of sar-miR172e (Additional file 17: Table S16), indicating that miR172 may participate in ABA-dependent and ABA-independent stress signaling during black seed germination through its targets. miR398 expression is induced by salt treatment in Populus [79] and T. salsuginea [72], but is repressed by oxidative stresses in Arabidopsis [80] and high levels of copper and cadmium in Medicago truncatula [81]. The targets of miR398 are $\mathrm{Cu} / \mathrm{Zn}$ superoxide dismutase (CSD) that can detoxify superoxide molecules. When copper supply is limited, 
the accumulation of miR398 reduces the allocation of copper into CSDs and saves copper for other essential processes [82, 83]. In S. aralocaspica, sar-miR398c was down-regulated in black seed while up-regulated in brown seed at S stage when comparing with the other two stages (Table 1, Additional file 16: Table S15). Although no unigene was predicted as the target of sarmiR398c in this study, we assumed that miR398 may play an important role in mediating the copper homeostasis that is required for photosynthetic and respiratory electron transport, oxidative stress protection, cell wall metabolism [84] during black and brown seed germination. Kinases and phosphatases have been documented to be involved in the regulation of proteins involved in osmolyte synthesis and detoxification by oxidants [54, 85]. They may play a role in salinity tolerance. In this study, a protein kinase superfamily protein was the candidate target of sar-miR5 (Additional file 18: Table S17). sar-miR5 was up-regulated in IS vs. DS and downregulated in $\mathrm{S}$ vs. IS in black seed, but was downregulated in IS vs. DS and up-regulated in S vs. IS in brown seed (Table 1, Additional file 16: Table S15), suggesting sar-miR5 may regulate salt tolerance through its target in diverse pathways in black and brown seed during S. aralocaspica germination. Wall associated kinaselike (WAKL) 1 and putative indole-3-acetic acid (IAA)amido synthetase GH3.9 were predicted to be the targets of sar-miR18 (Additional file 18: Table S17). WAKL members respond to environmental stresses and are developmentally regulated and tissue specific [86]. In Arabidopsis, WAKL1 has highest expression level in roots [86]. GH3.9 functions as IAA-amido synthetase to conjugate amino acids to the plant hormone auxin. gh3.9-1 mutants had greater primary root length, and increased sensitivity to IAA-mediated root growth inhibition [87]. At present study, sar-miR18 was down-regulated at S stage comparing to DS stage in brown seed (Table 1, Additional file 16: Table S15), while no significant sarmiR18 expression difference was identified between the germination stages in black seed, implying sar-miR18 maybe involved in environmental stresses response and root growth during germination and early seedling growth of brown seed by regulating WAKL1 and GH3.9 genes.

\section{Candidate miRNAs that may be related to the cautious germination strategy of black seed}

In plants, stem cells positioned in shoot apical meristem (SAM) and root apical meristem (RAM) constitute a pool of undifferentiated cells that continually provides new cells for post-embryonic growth [88]. miR166/165 have a conserved role in the maintenance of shoot and root apical meristems activity by negatively regulating its target, CLASS III HOMEODOMAIN-LEUCINE ZIPPER
(HD-ZIP III) [89-91]. In S. aralocaspica, sar-miR166b$3 \mathrm{p} / \mathrm{d} / \mathrm{l} / \mathrm{r}$ were down-regulated in black seed at $\mathrm{S}$ stage comparing to the other two stages (Table 1, Additional file 16: Table S15), while maintained at a high level in brown seed. Three HD-ZIP III transcription factors were predicted as the targets of sar-miR166b-3p/l/r, and one HD-ZIP III transcription factor was predicted to be the target of sar-miR166d (Table 2, Additional file 17: Table S16). This finding indicated that black seed may have a lower meristem activity than brown seed at seedling stage. The down-regulation of sar-miR166b-3p/d/l/r at $S$ stage could be a cautious germination strategy for black seed to respond quickly and proactively to the precarious environment with low risk to seedling survival.

\section{Conclusions}

In this study, we performed a systematic analysis of genes and miRNAs in S. aralocaspica. Our data revealed that specific genes and miRNAs were regulated differently between black and brown seed during germination. These candidate genes/miRNAs may contribute to the different germination behaviors of $S$. aralocaspica dimorphic seeds under the control of a bet-hedging strategy. This study elucidated the molecular mechanisms underlying the control of the timing of S. aralocaspica germination, stress tolerance during dimorphic seed germination, and the cautious germination strategy of black seed. The findings of this study provided a solid foundation for further understanding of the heteromorphic seed germination of halophytes in desert regions.

\section{Methods}

\section{Plant materials}

Freshly matured fruits of Suaeda aralocaspica were collected from plants in a natural population $\left(44^{\circ} 14^{\prime} \mathrm{N}\right.$; $87^{\circ} 44^{\prime}$ E; $445 \mathrm{~m}$ a.s.l) growing at the Fukang Desert Ecosystem Observation and Experimental Station in Xinjiang Province, China in early October 2013. The specimens used in this study were not deposited in a herbarium. Fruits were dried naturally for ten days under ambient room conditions. After that, seeds were separated from the dried plant material and sorted into black and brown seeds. S. aralocaspica black and brown dry seeds were sown on two layers of Whatman paper soaked with distilled water and incubated in a cabinet at $25{ }^{\circ} \mathrm{C}$ with continuous light. Imbibed brown seed were harvested $1 \mathrm{~h}$ after sowing, seedlings from brown seeds were harvested $24 \mathrm{~h}$ after sowing. Imbibed black seed were harvested $24 \mathrm{~h}$ after sowing, seedlings from black seeds were harvested within $10 \mathrm{~d}$ after sowing. Collected samples were frozen in liquid nitrogen and stored at $-80{ }^{\circ} \mathrm{C}$ for further analysis. 


\section{CDNA library preparation and RNA-seq}

Total RNA was extracted by using TRIzol $^{\circ}$ reagent (Invitrogen, Carlsbad, CA, USA) according to the manufacturer's instructions, and RQ1 DNase (Promega, Madison, WI, USA) was used to remove contaminating genomic DNA. The quality and quantity of the purified RNA was monitored at the ratios of A260/A280 and A260/230 on SmartSpec Plus Spectrophotometer (BioRad, Philadelphia, PA, USA). RNA integrity was further verified by $1.5 \%$ agarose gel electrophoresis and assessed by Agilent 2100 Bioanalyzer (Agilent Technologies, Santa Clara, CA, USA).

Equal amounts of RNA isolated from the samples collected at the same stages (DS, IS, S) were mixed together to prepare the cDNA library. mRNAs were purified and concentrated with Magnetic Beads Oligo (dT) (Invitrogen, Carlsbad, CA, USA). Purified mRNAs were iron fragmented at $95{ }^{\circ} \mathrm{C}$ followed by end repair and $5^{\prime}$ adaptor ligation. Then, reverse transcription was performed with RT primer harboring 3' adaptor sequence and randomized hexamer. Six cDNA libraries with insert sizes from 300 to 500 bp were prepared for Illumina HiSeq 2000 system $101 \mathrm{nt}$ pair-end sequencing.

\section{RNA-seq data filter}

We removed the low-quality reads with these criteria: 1 ) raw reads containing more than $2-\mathrm{N}$ bases were removed, 2) the reads were processed by clipping adaptor, 3) low quality bases were removed, 4) too short reads (less than $16 \mathrm{nt}$ ) were removed. FASTX-Toolkit [92] (Version 0.0.13) was used to filter the raw reads. All RNA sequencing reads were deposited to NCBI under BioProject accession number PRJNA325861.

\section{Assembly and statistics}

We used Trinity [16] with a 25-mer parameter for de novo assembly of the clean reads to generate a nonredundant set of transcripts, other default parameters including: group_pairs_distance $=500$, path_reinforcement_distance $=70$, min_kmer_cov $=1$. Afterwards, we realigned all clean reads onto the transcripts using Bowtie 2 [19] (Version 2.2.9), allowing up to four-base mismatches. We calculated the read coverage of each transcript. A transcript was defined not to be false positive, if the read coverage (the depth at least one read) was over $90 \%$ of the transcript.

After Trinity assembly, we used CD-HITv4.6.4-20150603 [20] for obtaining distinct sequences (transcripts). The following parameters were used to ensure quality of assembly: 1) sequence identity threshold: $0.95,2$ ) alignment coverage for the shorter sequence: 0.9, 3) maximum unmatched percentage (excluding leading and tailing gaps) for the shorter sequence must not be more than $10 \%$ of the sequence.

\section{Annotation and predicted CDS}

All assembled transcripts were searched against $\mathrm{Nr}$, COG, and Swiss-Prot protein database with BLASTX althorithm [93], and KEGG by BLAST2GO [21]. The Evalue cut-off was set to $10^{-5}$. Genes were identified according to best hits against known sequence functions, prediction of GO terms were also performed. The CDS were selected from transcript sequences based on the above alignment results, and transcripts not uncovered in the results were predicted by ESTScan [94]. The shortest CDS were at least $100 \mathrm{bp}$.

In the meanwhile, transcripts were annotated by $B$. vulgaris genome [95] and P. euphratica genome [96] and their whole proteome using BLAT [97] and BLASTX [93] (E-value $\left.<10^{-5}\right)$, respectively.

\section{Differential expression analysis of unigenes}

All clean reads were realigned onto the assembled transcripts using Bowtie 2 [19], allowing up to four-base mismatches. Trinity is able to report all alternatively spliced isoforms and transcripts derived from paralogous genes [16]. In order to calculate the read number and RPKM value for each gene, the alternative isoforms or paralogous transcripts were merged as one gene, the reads aligned with more than one gene were discarded due to their ambiguous location. Uniquely localized reads were used to calculate read number and RPKM value for each gene. To determine the differentially expressed unigenes between any two germination stages of $S$. aralocaspica, gene expression level analysis was performed using EdgeR package [22]. For each gene, the $p$-value and FDR were obtained based on the model of negative binomial distribution. The fold change (FC) of expression was also calculated within EdgeR. $\left|\log _{2} \mathrm{FC}\right|$ $\geq 1$ and $p$-value $<0.01$ were set as the threshold to define DEGs. Identified DEGs were further annotated using BLASTX [93] against $\mathrm{Nr}$ and Arabidopsis Information Resource (TAIR) database with a cut-off E-value of $10^{-5}$.

\section{sRNA library construction}

Five to six samples collected at each germination stage were mixed together, total RNA was extracted from the mixture. Three $\mu \mathrm{g}$ of each RNA sample was used for sRNA cDNA library preparation with Balancer NGS Library Preparation Kit (GnomeGen, San Diego, CA, USA) based on manufacturer's instruction. Whole library was applied to $10 \%$ native PAGE gel electrophoresis and bands corresponding to miRNA insertion were cut and eluted. After ethanol precipitation and washing, the purified small RNA libraries were quantified with Qubit Fluorometer (Invitrogen, Carlsbad, CA, USA) and used for cluster generation and applied to Illumina GAIIx (Illumina, San Diego, CA, USA) 73 nt and Illumina HiSeq $2000100 \mathrm{nt}$ single-end sequencing. 
Bioinformatic analysis of the sRNA transcriptome

All sequencing data was processed by FASTX-Toolkit [92], adaptor sequences and low quality tags were filtered. Based on the length of the mature miRNA and adaptor length, sequences shorter than $18 \mathrm{nt}$ and greater than $30 \mathrm{nt}$ in length were removed. At this step, the data were screened for redundant sequences. The remaining sequences were mapped to Rfam database (version 11.0 [98]) and S. aralocaspica mRNA transcriptome database for perfect matches, using custom-written PERL script. The matches to rRNAs, tRNAs or mRNAs were excluded. To identify conserved miRNAs, the retained unique sequences were aligned against miRBase (version 21), which contains 8582 miRNAs across 75 plant species [99], and the newly identified 241 miRNAs in Salicornia europaea [33], using Bowtie 2 [19] (one mismatch allowed). Only the perfectly matched sRNA sequences were considered to be conserved miRNAs. To reveal conserved and novel miRNA precursors, the unique sequences that have 10 or more counts were aligned to the $S$. aralocaspica mRNA transcriptome database using miRDeep-P with default parameters [100]. BLASTX [93] was used to match the sequences to $\mathrm{Nr}$ database, the putative precursors conserved in other plant species were removed. Mfold [31] was used to predict the secondary structures of the putative precursors utilizing default parameters. The miRNA precursors should met the following criteria: 1) forming an appropriate stem-loop structure, with a mature miRNA sitting in one arm of the hairpin structure; 2) mature miRNAs had no more than 6 mismatches with the opposite miRNA sequences; 3 ) the minimal folding free energy (MFE) of the hairpin structure was less than $-15 \mathrm{kcal} / \mathrm{mol}$; 4) MFE index were more than 0.5 ; 5) $\mathrm{A}+\mathrm{U}$ content was between 30 and $70 \%[33,101]$.

All small RNA sequencing reads were deposited to NCBI under BioProject accession number PRJNA325861.

\section{Differential expression analysis of miRNAs}

All clean reads from sRNA libraries were aligned against the mature sequences of identified miRNAs (only one mismatch allowed), the mapped reads were normalized to tags per million (TPM). Differentially expressed miRNAs between the three stages, DS, IS, S, were analyzed using Fisher Exact Test [102], and the mapped reads were pre-normalized by the locally weighted scatter plot smoothing method. $p$-value $<0.01$ and $\left|\log _{2} \mathrm{FC}\right| \geq 1$ were set as the threshold to define DE miRNAs.

\section{miRNA target prediction and validation}

The potential miRNA targets were identified using TAPIR [32] with default settings. All predicted target transcripts were evaluated by scoring system and considered to be miRNA targets if TAPIR score was less than
3.5 and miRNA-transcript duplex free energy ratio (mfe_ratio) was more than 0.7 .

Two $\mu \mathrm{g}$ total RNA from equally mixed six RNA extractions of DS, IS, and $S$ was used to synthesize $5^{\prime}$ RACE-ready cDNAs with the $5^{\prime}$-Full RACE Kit (Takara Bio Inc., Otsu, Shiga, Japan) according to the manufacturer's instructions. The final PCR product was extracted and purified from a $2 \%$ agarose gel, cloned into pEASYT1 Vector (Beijing TransGen Biotech Co., Ltd., Beijing, China), and plasmid DNA from ten to twelve different colonies was sequenced. The outer and inner gene specific primers were listed in Additional file 24: Table S21.

\section{qRT-PCR}

One microgram of total RNA was reverse transcribed using M-MLV Reverse Transcriptase according to the manufacturer's protocol (Promega, Madison, WI, USA). We selected 18 s rRNA and U6 snRNA as the endogenous controls. The primers for examined unigenes were designed by us from the $S$. aralocaspica transcriptome sequences and optimized for PCR (Additional file 24: Table S21), and bulge-loop qRT-PCR primers for mature miRNAs and U6 were designed and provided by RIBOBIO (Guangzhou RIBOBIO Co., Ltd., Guangzhou, China). Real-time monitoring of PCR was performed with ABI3700 (Applied Biosystems, Grand Island, NY, USA) and TransStart Top Green qPCR SuperMix (TransGen Biotech, Beijing, China). Reaction was performed at $95{ }^{\circ} \mathrm{C}$ for $10 \mathrm{~min}$, and then cycled at $95{ }^{\circ} \mathrm{C}$ for $15 \mathrm{~s}, 60{ }^{\circ} \mathrm{C}$ for $60 \mathrm{~s}$ for 40 cycles. Each assay was performed in triplicate, real-time qRT-PCR data were analyzed based on $2^{-\Delta \Delta C t}$ method [103].

\section{Additional files}

Additional file 1: Figure S1. Morphology of seed germination in Suaeda aralocaspica. Bar $=1 \mathrm{~mm}$. (PDF $13983 \mathrm{~kb}$ )

Additional file 2: Table S1. Statistics of RNA sequencing reads. Table S2. Summary of de novo sequence assembly using Trinity. Table S3. Annotation on assembled transcripts by different public databases. Table S11. Statistics of small RNA sequences from the six small RNA libraries. (DOCX 59 kb)

Additional file 3: Figure S2. GO functional classification of Suaeda aralocaspica unigenes within the category of cellular component (A), biological process (B) and molecular function (C). The number of unigenes enriched in each subcategory is indicated above each bar. (PDF 406 kb)

Additional file 4: Figure S3. Length distribution of Suaeda aralocaspica assembled transcripts and coding region sequences (CDS) of the transcripts. nt, nucleotides. (PDF $507 \mathrm{~kb}$ )

Additional file 5: Table S4. Unigene RPKM value and annotation against Nr database at NCBI. (XLSX 5600 kb)

Additional file 6: Table S5. Unigenes differentially expressed during Suaeda aralocaspica seed germination. (XLSX 3688 kb)

Additional file 7: Table S6. Annotation of unigenes differentially expressed during Suaeda aralocaspica seed germination compared with KEGG database. (XLSX $67 \mathrm{~kb}$ ) 
Additional file 8: Table S7. Unigenes differentially expressed between black and brown dry seed. (XLSX $270 \mathrm{~kb}$ )

Additional file 9: Table S8. Annotation of unigenes differentially expressed between black and brown dry seed compared with KEGG database. (XLSX $27 \mathrm{~kb})$

Additional file 10: Table S9. Identification of unigenes involved in abscisic acid and gibberellic acid signal transduction. (XLSX 17 kb)

Additional file 11: Table S10. Identification of differentially expressed unigenes involved in circadian rhythm-plant pathway during black seed germination. (XLSX $12 \mathrm{~kb}$ )

Additional file 12: Table S12. Identification of conserved miRNAs in Suaeda aralocaspica. (XLSX 76 kb)

Additional file 13: Table S13. Identification of novel miRNAs in Suaeda aralocaspica. (XLSX $35 \mathrm{~kb}$ )

Additional file 14: Table S14. The precursor sequences of Suaeda aralocaspica miRNAs. (XLSX $32 \mathrm{~kb}$ )

Additional file 15: Figure S4. The secondary structures of Suaeda aralocaspica miRNAs predicted by Mfold. The mature sequences of miRNA were highlighted in yellow. (PDF $2399 \mathrm{~kb}$ )

Additional file 16: Table S15. miRNAs differentially expressed during Suaeda aralocaspica seed germination. (XLSX 57 kb)

Additional file 17: Table S16. The putative targets of Suaeda aralocaspica conserved miRNAs predicted by TAPIR. (XLSX $49 \mathrm{~kb}$ )

Additional file 18: Table S17. The putative targets of Suaeda aralocaspica novel miRNAs predicted by TAPIR. (XLSX $41 \mathrm{~kb}$ )

Additional file 19: Figure S5. Validation of the expression profiles of selected unigenes (A) and miRNAs (B). The scatterplot of unigene and miRNA expression shows the positive correlation between transcriptome data and real-time qRT-PCR results. (PDF $110 \mathrm{~kb}$ )

Additional file 20: Table S18. Expression profiles of unigenes/miRNAs measured by RNA/small RNA sequencing and real-time qRT-PCR. (XLSX 54 kb)

Additional file 21: Figure S6. Real-time $\mathrm{QRT}-\mathrm{PCR}$ validation of putative target genes at different germination stages. BIDS represents black dry seed, BIIS represents black imbibed seed, BIS represents seedlings germinated from black seed, BrDS represents brown dry seed, BrIS represents brown imbibed seed, BrS represents seedlings germinated from brown seed. (PDF $208 \mathrm{~kb}$ )

Additional file 22: Table S19. Unigenes involved in photosynthesisantenna proteins pathway identified by KEGG enrichment. (XLSX $12 \mathrm{~kb}$ )

Additional file 23: Table S20. Unigenes involved in photosynthesis pathway identified by KEGG enrichment. (XLSX 56 kb)

Additional file 24: Table S21. The primers used in this study. (XLSX $10 \mathrm{~kb})$

\section{Abbreviations}

ABA: Abscisic acid; BI: Black; Br: Brown; CDS: Coding region sequences; COG: Clusters of orthologous groups; DE: Differentially expressed; DEG: Differentially expressed genes; DS: Dry seed; FC: Fold change; GA: Gibberellic acid; GO: Gene ontology; IS: Imbibed seed; KEGG: Kyoto encyclopedia of genes and genome; MFE: Minimal folding free energy; mfe_ratio: miRNA-transcript duplex free energy ratio; MFEl: Minimal folding free energy indice; Nr: Non-redundant; qRT-PCR: Real-time quantitative reverse transcription-PCR; RACE: Rapid amplification of CDNA ends; RNAseq: RNA sequence; RPKM: The reads per kilobase of transcript per million mapped reads; S: Seedling; sRNA-seq: Small RNA sequence; TAIR: Arabidopsis information resource; TPM: The mapped reads were normalized to tags per million

\section{Acknowledgements}

Not applicable.

\section{Funding}

This study was supported by the National Natural Science Foundation of China (31100295), State Key Laboratory of Desert and Oasis Ecology (Y371162), and Youth Innovation Promotion Association, Chinese Academy of Sciences (2013280). National Natural Science Foundation of China
(31100295) and Youth Innovation Promotion Association, Chinese Academy of Sciences (2013280) provided support in the form of salaries for author LW, but did not have any additional role in the design of the study and collection, analysis, and interpretation of data and in writing the manuscript. State Key Laboratory of Desert and Oasis Ecology (Y371162) provided support in the form of refunds to author LW who conducted scientific research in State Key Laboratory of Desert and Oasis Ecology, but did not have any additional role in the design of the study and collection, analysis, and interpretation of data and in writing the manuscript.

\section{Availability of data and materials}

The datasets that support the findings of this article are available at NCBI with BioProject ID: PRJNA325861 (http://www.ncbi.n/m.nih.gov/bioproject/ 325861) [104]. The release date is the date that the data is published.

\section{Authors' contributions}

LW, LY and $C T$ made substantial contributions to conception and design of the experiments. LW, HW and LY made substantial contributions to acquisition of data. LW, HW, LY and CT made substantial contributions to analysis and interpretation of data. LW and HW performed the validation experiments. LW and LY were involved in drafting the manuscript. HW, LY and $C T$ revised the manuscript critically for important intellectual content Each author participated sufficiently in the work to take public responsibility for appropriate portions of the content. All authors read and approved the final manuscript. All authors agreed to be accountable for all aspects of the work in ensuring that questions related to the accuracy or integrity of any part of the work are appropriately investigated and resolved.

\section{Ethics approval and consent to participate}

Freshly matured fruits of Suaeda aralocaspica were collected from plants in a natural population ( $44^{\circ} 14^{\prime} \mathrm{N} ; 87^{\circ} 44^{\prime} \mathrm{E} ; 445 \mathrm{~m}$ a.s.l) growing at the Fukang Desert Ecosystem Observation and Experimental Station in Xinjiang Province, China in early October 2013. Fruits were dried naturally for ten days under ambient room conditions. After that, seeds were separated from the dried plant material and sorted into black and brown seeds. S. aralocaspica is a plant species that is not listed under Convention on International Trade in Endangered Species of Wild Fauna and Flora (https://cites.org/eng/app/ appendices.php). No ethics approval is required for collection of $S$. aralocaspica samples or performing experiments on S. aralocaspica samples.

\section{Consent for publication}

Not applicable.

\section{Competing interests}

The authors declare that they have no competing interests.

\section{Publisher's Note}

Springer Nature remains neutral with regard to jurisdictional claims in published maps and institutional affiliations.

Received: 18 April 2017 Accepted: 12 October 2017

Published online: 19 October 2017

\section{References}

1. Gutterman Y. Seed germination in desert plants. Springer-Verlag Berlin Heidelberg; 1993.

2. Gutterman Y. Survival strategies of annual desert plants. Springer-Verlag Berlin Heidelberg; 2002.

3. Wang L, Huang Z, Baskin CC, Baskin JM, Dong M. Germination of dimorphic seeds of the desert annual halophyte Suaeda aralocaspica (Chenopodiaceae), a C4 plant without Kranz anatomy. Ann Bot. 2008;102: 757-69.

4. Baskin JM, Baskin CC. A classification system for seed dormancy. Seed Sci Res. 2004;14:1-16.

5. Hilhorst HW. A critical update on seed dormancy. I. Primary dormancy. Seed Sci Res. 1995:5:61-73.

6. Bewley JD. Seed germination and dormancy. Plant Cell. 1997;9:1055.

7. Li B, Foley ME. Genetic and molecular control of seed dormancy. Trends Plant Sci. 1997;2:384-9.

8. Venable DL. The evolutionary ecology of seed heteromorphism. Am Nat. 1985:577-95. 
9. Khan MA, Gul B. High salt tolerance in germinating dimorphic seeds of Arthrocnemum Indicum. Int J Plant Sci. 1998:826-32.

10. Wei $Y$, Dong M, Huang Z-Y. seed polymorphism, dormancy and germination of Salsola Affinis (Chenopodiaceae), a dominant desert annual inhabiting the Junggar Basin of Xinjiang, China. Aust J Bot. 2007:55:464-70.

11. Nakabayashi K, Okamoto M, Koshiba T, Kamiya Y, Nambara E. Genome-wide profiling of stored mRNA in Arabidopsis Thaliana seed germination: epigenetic and genetic regulation of transcription in seed. Plant J. 2005;41:697-709.

12. Preston J, Tatematsu K, Kanno Y, Hobo T, Kimura M, Jikumaru Y, Yano R, Kamiya $Y$, Nambara E. Temporal expression patterns of hormone metabolism genes during imbibition of Arabidopsis Thaliana seeds: a comparative study on dormant and non-dormant accessions. Plant Cell Physiol. 2009:50:1786-800.

13. Narsai R, Law SR, Carrie C, Xu L, Whelan J. In-depth temporal transcriptome profiling reveals a crucial developmental switch with roles for RNA processing and organelle metabolism that are essential for germination in Arabidopsis. Plant Physiol. 2011;157:1342-62.

14. Endo A, Tatematsu K, Hanada K, Duermeyer L, Okamoto M, YonekuraSakakibara K, Saito K, Toyoda T, Kawakami N, Kamiya Y. Tissue-specific transcriptome analysis reveals cell wall metabolism, flavonol biosynthesis and defense responses are activated in the endosperm of germinating Arabidopsis Thaliana seeds. Plant Cell Physiol. 2012;53:16-27.

15. Penfield S, Li Y, Gilday AD, Graham S, Graham IA. Arabidopsis ABA INSENSITIVE4 regulates lipid mobilization in the embryo and reveals repression of seed germination by the endosperm. Plant Cell. 2006;18:1887-99.

16. Grabherr MG, Haas BJ, Yassour M, Levin JZ, Thompson DA, Amit I, Adiconis X, Fan L, Raychowdhury R, Zeng Q. Full-length transcriptome assembly from RNA-Seq data without a reference genome. Nat Biotechnol. 2011;29:644-52.

17. Liu S, Li W, Wu Y, Chen C, Lei J. De novo transcriptome assembly in chili pepper (Capsicum Frutescens) to identify genes involved in the biosynthesis of capsaicinoids. PLoS One. 2013. doi:10.1371/journal.pone.0048156.

18. Wu T, Luo S, Wang R, Zhong $Y, X u X$. Lin ye, he $X$, sun $B$, Huang $H$ : the first Illumina-based de novo transcriptome sequencing and analysis of pumpkin (Cucurbita Moschata Duch.) and SSR marker development. Mol Breed. 2014; 34:1437-47.

19. Langmead B, Salzberg SL. Fast gapped-read alignment with bowtie 2. Nat Methods. 2012;9:357-9.

20. Fu L, Niu B, Zhu Z, Wu S, Li W. CD-HIT: accelerated for clustering the nextgeneration sequencing data. Bioinformatics. 2012;28:3150-2.

21. Conesa A, Götz S, García-Gómez JM, Terol J, Talón M, Robles M. Blast2GO: a universal tool for annotation, visualization and analysis in functional genomics research. Bioinformatics. 2005;21:3674-6.

22. Robinson MD, McCarthy DJ, Smyth GK. edgeR: a bioconductor package for differential expression analysis of digital gene expression data. Bioinformatics. 2010;26:139-40.

23. Yuan K, Rashotte AM, Wysocka-Diller JW. ABA and GA signaling pathways interact and regulate seed germination and seedling development under salt stress. Acta Physiol Plant. 2011;33:261-71.

24. Weitbrecht K, Müller K, Leubner-Metzger G. First off the mark: early seed germination. J Exp Bot. 2011;62:3289-309.

25. Rajjou L, Duval M, Gallardo K, Catusse J, Bally J, Job C, Job D. Seed germination and vigor. Annu Rev Plant Biol. 2012;63:507-33.

26. Penfield $\mathrm{S}$, Hall A. A role for multiple circadian clock genes in the response to signals that break seed dormancy in Arabidopsis. Plant Cell. 2009;21:1722-32.

27. Covington MF, Maloof JN, Straume M, Kay SA, Harmer SL. Global transcriptome analysis reveals circadian regulation of key pathways in plant growth and development. Genome Biol. 2008;9:1.

28. Michael TP, Breton G, Hazen SP, Priest H, Mockler TC, Kay SA, Chory J. A morning-specific phytohormone gene expression program underlying rhythmic plant growth. PLoS Biol. 2008. doi:10.1371/journal.pbio.0060225.

29. Henderson IR, Zhang X, Lu C, Johnson L, Meyers BC, Green PJ, Jacobsen SE. Dissecting Arabidopsis Thaliana DICER function in small RNA processing, gene silencing and DNA methylation patterning. Nat Genet. 2006:38:721-5.

30. Zhang B, Pan X, Cox S, Cobb G, Anderson T. Evidence that miRNAs are different from other RNAs. Cell Mol Life Sci. 2006;63:246-54.

31. Zuker M. Mfold web server for nucleic acid folding and hybridization prediction. Nucleic Acids Res. 2003;31:3406-15.

32. Bonnet $E$, He $Y$, Billiau $K$, Van de Peer $Y$. TAPIR, a web server for the prediction of plant microRNA targets, including target mimics. Bioinformatics. 2010;26:1566-8.
33. Feng J, Wang J, Fan P, Jia W, Nie L, Jiang P, Chen X, Lv S, Wan L, Chang S. High-throughput deep sequencing reveals that microRNAs play important roles in salt tolerance of euhalophyte Salicornia Europaea. BMC Plant Biol. 2015;15:1.

34. Ronemus M, Vaughn MW, Martienssen RA. MicroRNA-targeted and small interfering RNA-mediated mRNA degradation is regulated by Argonaute, Dicer, and RNA-dependent RNA polymerase in Arabidopsis. Plant Cell. 2006; 18:1559-74.

35. Wan L-C, Zhang H, Lu S, Zhang L, Qiu Z, Zhao Y, Zeng Q-Y, Lin J. Transcriptome-wide identification and characterization of miRNAs from Pinus Densata. BMC Genomics. 2012:13:132.

36. Rolletschek H, Radchuk R, Klukas C, Schreiber F, Wobus U, Borisjuk L. Evidence of a key role for photosynthetic oxygen release in oil storage in developing soybean seeds. New Phytol. 2005;167:777-86.

37. Rolletschek $\mathrm{H}$, Weber $\mathrm{H}$, Borisjuk L. Energy status and its control on embryogenesis of legumes. Embryo photosynthesis contributes to oxygen supply and is coupled to biosynthetic fluxes. Plant Physiol. 2003;132:1196-206.

38. Ruuska SA, Schwender J, Ohlrogge JB. The capacity of green oilseeds to utilize photosynthesis to drive biosynthetic processes. Plant Physiol. 2004; 136:2700-9.

39. Brown DE, Rashotte AM, Murphy AS, Normanly J, Tague BW, Peer WA, Taiz L, Muday GK. Flavonoids act as negative regulators of auxin transport in vivo in Arabidopsis. Plant Physiol. 2001;126:524-35.

40. Buer CS, Djordjevic MA. Architectural phenotypes in the transparent testa mutants of Arabidopsis Thaliana. J Exp Bot. 2009;60:751-63.

41. Buer CS, Muday GK. The transparent testa4 mutation prevents flavonoid synthesis and alters auxin transport and the response of Arabidopsis roots to gravity and light. Plant Cell. 2004;16:1191-205.

42. Lewis DR, Ramirez MV, Miller ND, Vallabhaneni P, Ray WK, Helm RF, Winkel BS, Muday GK. Auxin and ethylene induce flavonol accumulation through distinct transcriptional networks. Plant Physiol. 2011;156:144-64.

43. Peer WA, Bandyopadhyay A, Blakeslee JJ, Makam SN, Chen RJ, Masson PH, Murphy AS. Variation in expression and protein localization of the PIN family of auxin efflux facilitator proteins in flavonoid mutants with altered auxin transport in Arabidopsis Thaliana. Plant Cell. 2004;16:1898-911.

44. Sabatini S, Beis D, Wolkenfelt H, Murfett J, Guilfoyle T, Malamy J, Benfey P, Leyser O, Bechtold N, Weisbeek P. An auxin-dependent distal organizer of pattern and polarity in the Arabidopsis root. Cell. 1999;99:463-72.

45. Dubrovsky JG, Sauer M, Napsucialy-Mendivil S, Ivanchenko MG, Friml J, Shishkova S, Celenza J, Benková E. Auxin acts as a local morphogenetic trigger to specify lateral root founder cells. Proc Natl Acad Sci. 2008;105: 8790-4.

46. Friml J, Benková E, Blilou I, Wisniewska J, Hamann T, Ljung K, Woody S, Sandberg G, Scheres B, Jürgens G. AtPIN4 mediates sink-driven auxin gradients and root patterning in Arabidopsis. Cell. 2002;108:661-73.

47. Pelletier MK, Shirley BW. Analysis of flavanone 3-hydroxylase in Arabidopsis seedlings (coordinate regulation with chalcone synthase and chalcone isomerase). Plant Physiol. 1996;111:339-45.

48. Cain CC, Saslowsky DE, Walker RA, Shirley BW. Expression of chalcone synthase and chalcone isomerase proteins in Arabidopsis seedlings. Plant Mol Biol. 1997;35:377-81.

49. Dharmasiri N, Dharmasiri S, Estelle M. The F-box protein TIR1 is an auxin receptor. Nature. 2005:435:441-5.

50. Dharmasiri N, Dharmasiri S, Weijers D, Lechner E, Yamada M, Hobbie L, Ehrismann JS, Jürgens G, Estelle M. Plant development is regulated by a family of auxin receptor F box proteins. Dev Cell. 2005;9:109-19.

51. Kepinski S, Leyser $\mathrm{O}$. The Arabidopsis F-box protein TIR1 is an auxin receptor. Nature. 2005;435:446-51.

52. Kepinski S, Leyser O. The Arabidopsis F-box protein TIR1 is an auxin receptor. Nature. 2005;435:446.

53. Lee YP, Giorgi FM, Lohse M, Kvederaviciute K, Klages S, Usadel B, Meskiene I, Reinhardt R, Hincha DK. Transcriptome sequencing and microarray design for functional genomics in the extremophile Arabidopsis relative Thellungiella Salsuginea (Eutrema Salsugineum). BMC Genomics. 2013;14:793.

54. Diray-Arce J, Clement M, Gul B, Khan MA, Nielsen BL. Transcriptome assembly, profiling and differential gene expression analysis of the halophyte Suaeda Fruticosa provides insights into salt tolerance. BMC Genomics. 2015;16:353.

55. Boudsocq $M$, Barbier-Brygoo $H$, Laurière $C$. Identification of nine sucrose nonfermenting 1-related protein kinases 2 activated by hyperosmotic and saline stresses in Arabidopsis Thaliana. J Biol Chem. 2004;279:41758-66. 
56. Yoshida R, Hobo T, Ichimura K, Mizoguchi T, Takahashi F, Aronso J, Ecker JR, Shinozaki K. ABA-activated SnRK2 protein kinase is required for dehydration stress signaling in Arabidopsis. Plant Cell Physiol. 2002:43:1473-83.

57. Piskurewicz U, Jikumaru Y, Kinoshita N, Nambara E, Kamiya Y, Lopez-Molina L. The gibberellic acid signaling repressor RGL2 inhibits Arabidopsis seed germination by stimulating abscisic acid synthesis and ABI5 activity. Plant Cell. 2008;20:2729-45.

58. Tyler L, Thomas SG, Hu J, Dill A, Alonso JM, Ecker JR, Sun T-P. DELLA proteins and gibberellin-regulated seed germination and floral development in Arabidopsis. Plant Physiol. 2004;135:1008-19.

59. Kurihara Y, Watanabe Y. Arabidopsis Micro-RNA biogenesis through Dicerlike 1 protein functions. Proc Natl Acad Sci U S A. 2004;101:12753-8.

60. Xie Z, Kasschau KD, Carrington JC. Negative feedback regulation of DicerLike1 in Arabidopsis by microRNA-guided mRNA degradation. Curr Biol. 2003:13:784-9.

61. Das SS, Karmakar P, Nandi AK, Sanan-Mishra N. Small RNA mediated regulation of seed germination. Front Plant Sci. 2015. doi:10.3389/fpls.2015. 00828.

62. Martin RC, Liu P-P, Goloviznina NA, Nonogaki H. microRNA, seeds, and Darwin?: diverse function of miRNA in seed biology and plant responses to stress. J Exp Bot. 2010;61:2229-34.

63. Martin RC, Asahina M, Liu P-P, Kristof JR, Coppersmith JL, Pluskota WE, Bassel GW, Goloviznina NA, Nguyen TT, Martínez-Andújar C. The regulation of post-germinative transition from the cotyledon-to vegetative-leaf stages by microRNA-targeted SQUAMOSA PROMOTER-BINDING PROTEIN LIKE13 in Arabidopsis. Seed Sci Res. 2010;20:89.

64. Martin RC, Asahina M, Liu P-P, Kristof JR, Coppersmith JL, Pluskota WE, Bassel GW, Goloviznina NA, Nguyen TT, Martínez-Andújar C. The microRNA156 and microRNA172 gene regulation cascades at postgerminative stages in Arabidopsis. Seed Sci Res. 2010;20:79-87.

65. Li D, Wang L, Liu X, Cui D, Chen T, Zhang H, Jiang C, Xu C, Li P, Li S. Deep sequencing of maize small RNAs reveals a diverse set of microRNA in dry and imbibed seeds. PLoS One. 2013;8:e55107.

66. Reyes $J$, Chua NH. ABA induction of miR159 controls transcript levels of two MYB factors during Arabidopsis seed germination. Plant J. 2007;49:592-606.

67. Stanga JP, Smith SM, Briggs WR, Nelson DC. SUPPRESSOR OF MORE AXILLARY GROWTH2 1 controls seed germination and seedling development in Arabidopsis. Plant Physiol. 2013;163:318-30.

68. Ma Z, Hu X, Cai W, Huang W, Zhou X, Luo Q, Yang H, Wang J, Huang J. Arabidopsis miR171-targeted scarecrow-like proteins bind to GT ciselements and mediate gibberellin-regulated chlorophyll biosynthesis under light conditions. PLoS Genet. 2014. doi:10.1371/journal.pgen.1004519.

69. Gao M-J, Li X, Huang J, Gropp GM, Gjetvaj B, Lindsay DL, Wei S, Coutu C, Chen Z, Wan X-C. SCARECROW-LIKE15 interacts with HISTONE DEACETYLASE19 and is essential for repressing the seed maturation programme. Nat Commun. 2015. doi:10.1038/ncomms8243.

70. Zhao B, Liang R, Ge L, Li W, Xiao H, Lin H, Ruan K, Jin Y. Identification of drought-induced microRNAs in rice. Biochem Biophys Res Commun. 2007; 354:585-90.

71. Zhao B, Ge L, Liang R, Li W, Ruan K, Lin H, Jin Y. Members of miR-169 family are induced by high salinity and transiently inhibit the NF-YA transcription factor. BMC Mol Biol. 2009;10:29.

72. Zhang Q, Zhao C, Li M, Sun W, Liu Y, Xia H, Sun M, Li A, Li C, Zhao S. Genome-wide identification of Thellungiella Salsuginea microRNAs with putative roles in the salt stress response. BMC Plant Biol. 2013;13:180.

73. Sorin C, Declerck M, Christ A, Blein T, Ma L, Lelandais-Brière C, Njo MF, Beeckman T, Crespi M, Hartmann C. A miR169 isoform regulates specific NF-YA targets and root architecture in Arabidopsis. New Phytol. 2014;202:1197-211.

74. Ni Z, Hu Z, Jiang Q, Zhang H. GmNFYA3, a target gene of miR169, is a positive regulator of plant tolerance to drought stress. Plant Mol Biol. 2013; 82:113-29.

75. Hundertmark $M$, Hincha DK. LEA (late embryogenesis abundant) proteins and their encoding genes in Arabidopsis Thaliana. BMC Genomics. 2008:9:118.

76. Nishimura N, Yoshida T, Kitahata N, Asami T, Shinozaki K, Hirayama T. ABAhypersensitive Germination1 encodes a protein phosphatase 2 , an essential component of abscisic acid signaling in Arabidopsis seed. Plant J. 2007;50:935-49.

77. Bhaskara GB, Nguyen TT, Verslues PE. Unique drought resistance functions of the highly ABA-induced clade a protein phosphatase 2Cs. Plant Physiol. 2012;160:379-95.

78. Karali D, Oxley D, Runions J, Ktistakis N, Farmaki T. The Arabidopsis Thaliana immunophilin ROF1 directly interacts with PI $(3)$ P and PI $(3,5)$ P 2 and affects germination under osmotic stress. PLoS One. 2012. doi:10.1371/ journal.pone.0048241.

79. Jia X, Wang W-X, Ren L, Chen Q-J, Mendu V, Willcut B, Dinkins R, Tang X, Tang G. Differential and dynamic regulation of miR398 in response to $A B A$ and salt stress in Populustremula and Arabidopsisthaliana. Plant Mol Biol. 2009;71:51-9.

80. Sunkar R, Kapoor A, Zhu J-K. Posttranscriptional induction of two cu/Zn superoxide dismutase genes in Arabidopsis is mediated by downregulation of miR398 and important for oxidative stress tolerance. Plant Cell. 2006;18: 2051-65.

81. Zhou ZS, Huang SQ, Yang ZM. Bioinformatic identification and expression analysis of new microRNAs from Medicago Truncatula. Biochem Biophys Res Commun. 2008;374:538-42.

82. Yamasaki H, Abdel-Ghany SE, Cohu CM, Kobayashi Y, Shikanai T, Pilon M. Regulation of copper homeostasis by micro-RNA in Arabidopsis. J Biol Chem. 2007;282:16369-78.

83. Abdel-Ghany SE, Pilon M. MicroRNA-mediated systemic down-regulation of copper protein expression in response to low copper availability in Arabidopsis. J Biol Chem. 2008;283:15932-45.

84. Marschner H. Mineral nutrition of higher plants. London: Academic Press; 1995.

85. Senadheera P, Maathuis FJ. Differentially regulated kinases and phosphatases in roots may contribute to inter-cultivar difference in rice salinity tolerance. Plant Signal Behav. 2009;4:1163-5.

86. Verica JA, Chae L, Tong H, Ingmire P, He Z-H. Tissue-specific and developmentally regulated expression of a cluster of tandemly arrayed cell wall-associated kinase-like kinase genes in Arabidopsis. Plant Physiol. 2003; 133:1732-46.

87. Khan S, Stone JM. Arabidopsis thalianaGH3. 9 influences primary root growth. Planta. 2007;226:21-34.

88. Wenig U, Meyer S, Stadler R, Fischer S, Werner D, Lauter A, Melzer M, Hoth S, Weingartner M, Sauer N. Identification of MAIN, a factor involved in genome stability in the meristems of Arabidopsis Thaliana. Plant J. 2013;75: 469-83.

89. Prigge MJ, Otsuga D, Alonso JM, Ecker JR, Drews GN, Clark SE. Class III homeodomain-leucine zipper gene family members have overlapping, antagonistic, and distinct roles in Arabidopsis development. Plant Cell. 2005; 17:61-76.

90. Williams L, Grigg SP, Xie M, Christensen S, Fletcher JC. Regulation of Arabidopsis shoot apical meristem and lateral organ formation by microRNA miR166g and its AtHD-ZIP target genes. Development. 2005;132: 3657-68.

91. Singh A, Singh S, Panigrahi KC, Reski R, Sarkar AK. Balanced activity of microRNA166/165 and its target transcripts from the class III homeodomainleucine zipper family regulates root growth in Arabidopsisthaliana. Plant Cell Rep. 2014;33:945-53.

92. Gordon A, Hannon G: Fastx-toolkit. FASTQ/A short-reads preprocessing tools (unpublished) http://hannonlab cshl edu/fastx_toolkit 2010,5.

93. Altschul SF, Gish W, Miller W, Myers EW, Lipman DJ. Basic local alignment search tool. J Mol Biol. 1990;215:403-10.

94. Iseli C, Jongeneel CV, Bucher P. ESTScan: a program for detecting, evaluating, and reconstructing potential coding regions in EST sequences. In: ISMB; 1999. p. 138-48.

95. Dohm JC, Minoche AE, Holtgräwe D, Capella-Gutiérrez S, Zakrzewski F, Tafer H, Rupp O, Sörensen TR, Stracke R, Reinhardt R. The genome of the recently domesticated crop plant sugar beet (Beta Vulgaris). Nature. 2014;505:546-9.

96. Ma T, Wang J, Zhou G, Yue Z, Hu Q, Chen Y, Liu B, Qiu Q, Wang Z, Zhang J. Genomic insights into salt adaptation in a desert poplar. Nat Commun. 2013. doi:10.1038/ncomms3797.

97. Kent WJ. BLAT—the BLAST-like alignment tool. Genome Res. 2002;12:656-64.

98. Burge SW, Daub J, Eberhardt R, Tate J, Barquist L, Nawrocki EP, Eddy SR, Gardner PP, Bateman A: Rfam 11.0: 10 years of RNA families. Nucleic acids research 2012:gks1005.

99. Kozomara A, Griffiths-Jones S. miRBase: annotating high confidence microRNAs using deep sequencing data. Nucleic Acids Res. 2014;42:D68-73.

100. Yang $X$, Li L. miRDeep-P: a computational tool for analyzing the microRNA transcriptome in plants. Bioinformatics. 2011;27:2614-5.

101. Wan L-C, Zhang H, Lu S, Zhang L, Qiu Z, Zhao Y, Zeng Q-Y, Lin J. Transcriptome-wide identification and characterization of miRNAs from Pinus Densata. BMC Genomics. 2012;13:1. 
102. Fisher RA. On the interpretation of $x 2$ from contingency tables, and the calculation of P. J R Stat Soc. 1922:85:87-94.

103. Livak KJ, Schmittgen TD. Analysis of relative gene expression data using real-time quantitative $P C R$ and the $2-\Delta \Delta C T$ method methods. 2001;25:402-8

104. Wang L, Wang $H$, Yin $L$, Tian C. Suaeda aralocaspica raw sequence reads. In: BioProject. National Center for Biotechnology Information. 2016. http://www.ncbi.nlm.nih.gov/bioproject/325861.

Submit your next manuscript to BioMed Central and we will help you at every step:

- We accept pre-submission inquiries

- Our selector tool helps you to find the most relevant journal

- We provide round the clock customer support

- Convenient online submission

- Thorough peer review

- Inclusion in PubMed and all major indexing services

- Maximum visibility for your research

Submit your manuscript at www.biomedcentral.com/submit 